\title{
The Influence of Composition, Distillated and Synthetic Sea Water and Elevated Temperature on Mechanical and Physical Characteristics of Four Cured Different Polyester Systems
}

\author{
Jovan Radulović1)
}

\begin{abstract}
In this paper the influence of composition of resins, distillated and synthetic sea water on mechanical and physical characteristics of four different polyester resin (PR) systems is presented. The basic data about chemistry of PR, ingredients of PR systems (polyhydric alcohols, an unsaturated polybasic acids, a saturated polybasic acids and monomer), process for manufacturing of hardened and post cured specimens and testing procedure are presented. Specimens of four PR systems were exposed to the influence of distillated and synthetic sea water at $23^{\circ} \mathrm{C}$ for a period of thirty days (the first experiment) and distillated boiling water $\left(100{ }^{\circ} \mathrm{C}\right)$ for a period of 210 minutes (the second experiment). Hardness, impact resistance, water soluble matter and water absorption of specimens of four $P R$ systems were recorded as the parameters that demonstrate the quality changes of tested materials in mentioned conditions of both experiments. It is established that ingredients and distillated and synthetic sea water have important influence on tested mechanical and physical characteristics of four PR systems.
\end{abstract}

Key words: polyester resin, mechanical properties, physical properties, distillated water, sea water, synthetic water, testing results.

\section{The list of abbreviations used in the paper}

\begin{tabular}{|c|c|}
\hline$m_{1}$ & - initial mass of a specimen, in $g$; \\
\hline$m_{2, x, d . w .}$ & $\begin{array}{l}\text { - mass of a specimen after immersion in } \\
\text { distillated water for } x \text { days, in } g ;\end{array}$ \\
\hline$m_{3, x, d . w ., c . m .}$ & $\begin{array}{l}\text { - mass of a specimen after immersion in } \\
\text { distillated water for } x \text { days and afterwards } \\
\text { dried in oven to constant mass, in } g \text {; }\end{array}$ \\
\hline$m_{2, x, \text { s.s.w. }}$ & $\begin{array}{l}\text { - mass of a specimen after immersion in } \\
\text { synthetic sea water for } x \text { days, in } g ;\end{array}$ \\
\hline$m_{3, x, s . s . w . c . m}$ & $\begin{array}{l}\text { - mass of a specimen after immersion in } \\
\text { synthetic sea water for } x \text { days and afterwards } \\
\text { dried in oven to constant mass, in } g \text {; }\end{array}$ \\
\hline$m_{2, y, b . w .}$ & $\begin{array}{l}\text { - mass of a specimen after immersion in } \\
\text { boiling water for } y \text { minutes, in } g \text {; }\end{array}$ \\
\hline$m_{3, y, b . w ., c . m}$ & $\begin{array}{l}\text { - mass of a specimen after immersion in } \\
\text { boiling water for } y \text { minutes and afterwards } \\
\text { dried in oven to constant mass, in } g \text {; }\end{array}$ \\
\hline H.S. & - specimen for hardness testing; \\
\hline I.R.S: & - specimen for impact resistance testing; \\
\hline$P R$ & - system - polyester resin system; \\
\hline D. $W$. & - distillated water; \\
\hline S.S.W & - synthetic sea water; \\
\hline B.D. & - Barcol degree. \\
\hline
\end{tabular}

\section{Introduction}

ECOND half of the previous century and decades of the $\checkmark$ current century up to today are considered as a period of intensive utilization of polymeric materials, although initial steps in development were recorded in the nineteenth century. Polyester resins have been one of mostly used polymeric thermosetting material in a long period of time for manufacturing very dissimilar things [1].

By chemical definition a polyester is formed by the reaction of a polybasic acid and a polyhydric alcohol to form a series (poly=many) of ester linkages.

The first attempt to make a polyester resin or an alkyd is attributed to J.J. Berzelius (Sweden) who, in 1847, reacted tartaric acid with glycerol to obtain a resinous mass. As early as 1916, resinous compositions, based on the combination of glycerol and phthalic anhydride were used as finishes for wood and metal. The combination of fatty acids from vegetable oils with alkyds by R.H. Kienle (USA), in 1927, was a major step toward useful products similar in compositions used in many of modern finishes. Kienle is given credit for coining the word ALKYD (derived from ALcohol-aCID and spelled phonetically). In 1937, Carleton Ellis discovered that the addition of unsaturated monomers to polyesters containing unsaturated groups increased their setting speed by more than thirty times. The combination of curing speed and properties of the resulting polymers gave the first thermosetting polyester resin which can be put in commercial use. For his key discoveries in the development of polyesters C. Ellis has sometimes been called the "father" of unsaturated polyesters [2].

\section{Chemistry of polyester resins}

In inorganic chemistry reaction between an acid and a base (so called neutralization reaction) produces salt and water.

A similar reaction involving organic molecules (based on carbon backbone) would be a reaction of organic acid and alcohol ("organic base") to yield an ester. For example, acetic acid and ethyl alcohol, in so called esterification or

\footnotetext{
1) Military Technical Institute (VTI), Ratka Resanovića 1, 11132 Belgrade, SERBIA

Correspondence to: Jovan Radulović; e-mail: jovan.r.radulovic@gmail.com
} 
condensation reaction, produced ethyl acetate and water.

Since the involved molecules are monobasic acid and monohydric alcohol, they have only one reactive group (each) and reaction stops with the formation of a "simple ester" (Fig.1) [2]:

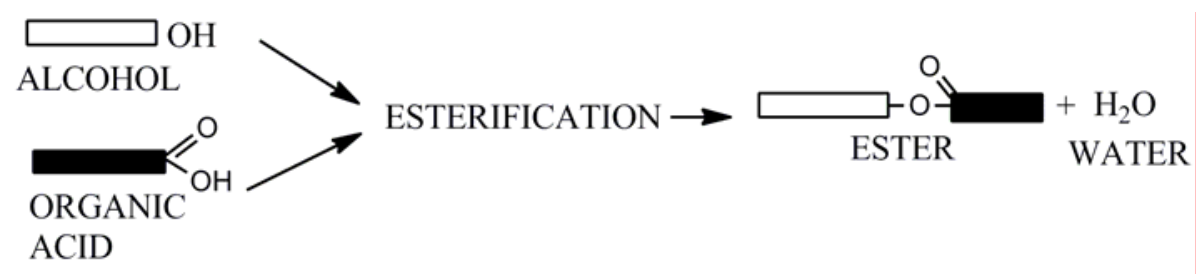

Figure 1. Simple esterification reaction

By functionality theory polybasic acid and polyhydric alcohol can react to form complex molecules or polymers. Thus a polybasic acid, as adipic acid, and polyhydric alcohol, as ethylene glycol, being bifunctional, can be esterified by multiple esterification reactions to yield a linear molecule of, theoretically, infinite length (Fig.2) [2]:

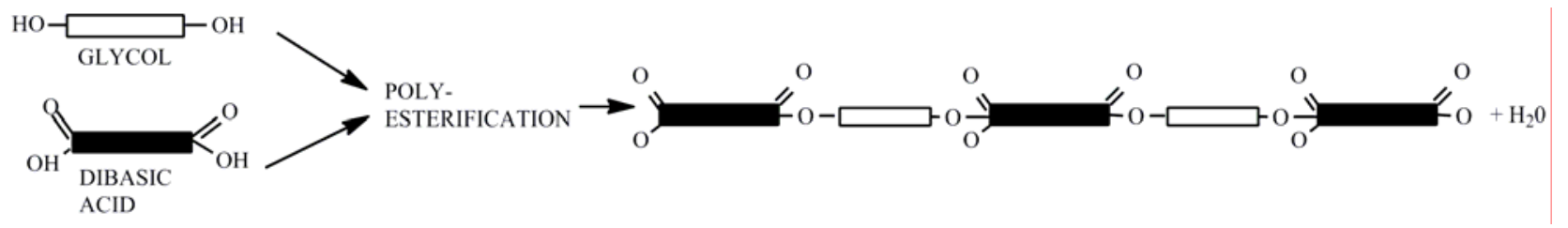

Figure 2. Polyesterification reaction

If either dibasic acid or dihydric alcohol contains unsaturated or double bonded $\mathrm{C}$ atoms, unsaturated polyester is obtained. The most frequently, in practice, unsaturated polybasic acid, saturated polybasic acid and polyhydric alcohol are used in polyesterification reactions (Fig.3) [2]:

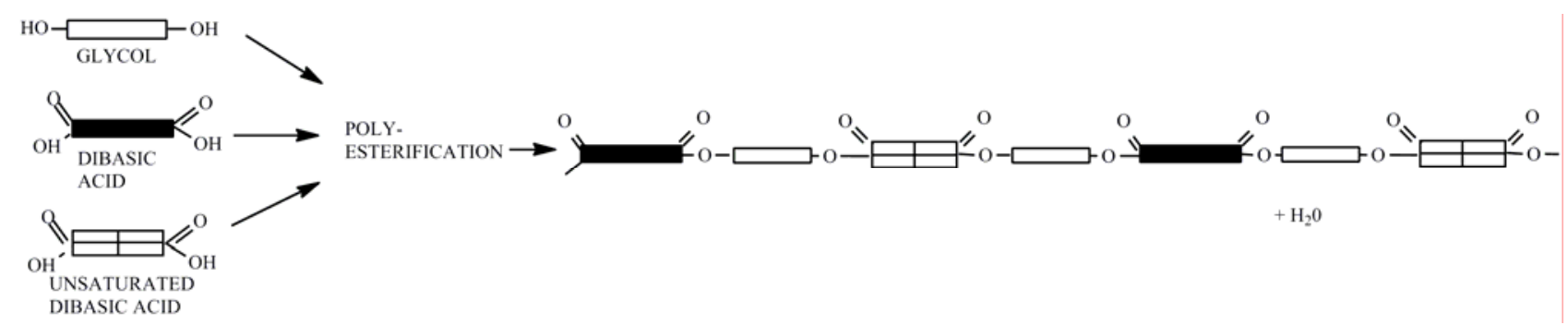

Figure 3. Polyesterification reaction between unsaturated dibasic acid, saturated dibasic acid and dihydric alcohol

Unsaturated polyester chain (produced by described manner), due to present double bonds, is capable of subsequent cross-linking or thermosetting either directly to similar double bonds in adjacent unsaturated polyester chain by direct reactions or, more frequently, through an unsaturated double bond in monomer (Fig.4) [2]:

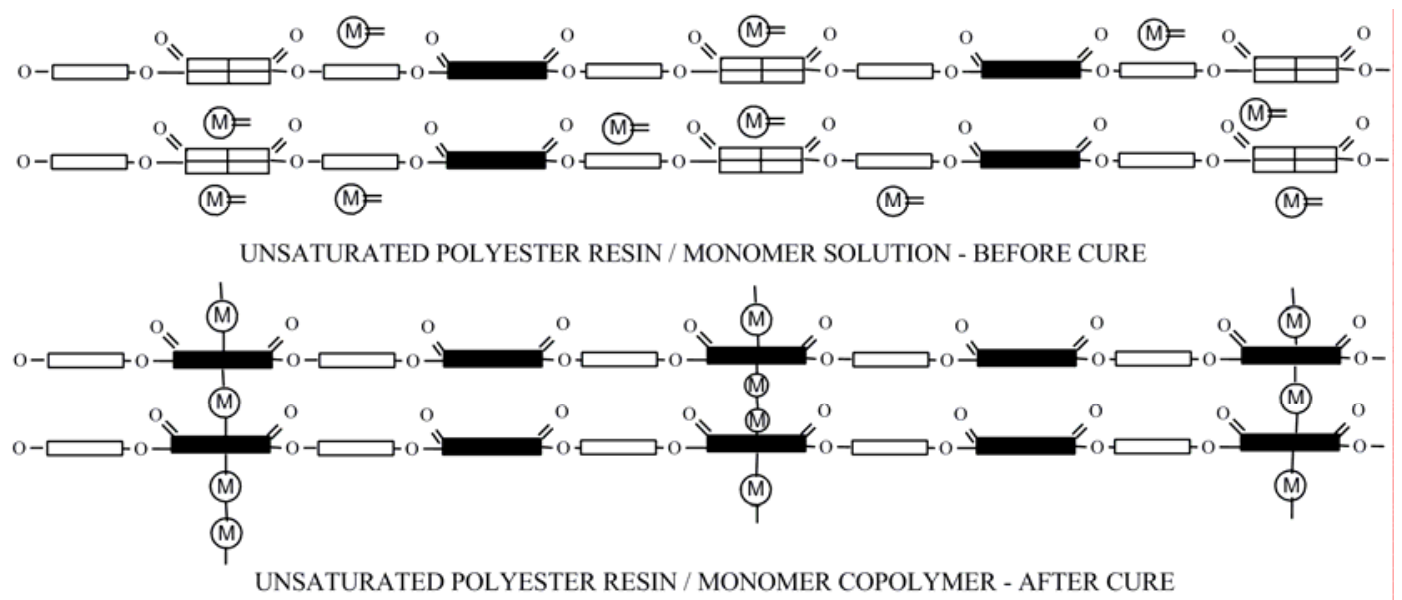

Figure 4. Unsaturated polyester/monomer mixture before and after cross-linking reaction

\section{Ingredients of polyester resin system}

Innumerable variations can be made in the composition of the base polyester and the type and amount of cross-linking monomer, to yield resins with a wide range of characteristics before, during and after polymerization.

To obtain a polymer chain which can be cross-linked into thermosetting resin, most often, a proportion of the dibasic acid must contain an unsaturated group or double bond. This unsaturation must be non-aromatic otherwise cross-linking will not take place. It is worth mentioned that styrene will react through the pendant vinyl group in the presence of suitable catalyst, whilst the benzene aromatic nucleus does not react. 
The types of acid selected-with respect to their configuration, pendant groups, and presence of unsaturated bonds available for cross-linking, molecular weight-contribute to the properties of the final product. Most of polyester resins are made up of at least two dibasic acids (mix of unsaturated and saturated acids), whilst a single unsaturated resin is only used where a high degree of cross-linking is desired. By varying the ratio of the two anhydrides or acids, the crosslinking reactivity of the polyester resin can be varied from high to low. A high reactivity polyester resin contains a high proportion of unsaturation.

Such a formed polymer could be converted into a thermoset by addition of catalyst (invariably peroxide). In order to convert this polymeric material into a usable resin, a further component, which acts as solvent for the polymer chain (i.e. viscosity reducer of the resin) and cross-linking agent, is necessary. Its component is monomer, which, during cure links the polymer chains together through the unsaturated reactive groups, to give a usable product [3].

Thus, it can be seen that there are four main components needed to produce a usable resin, namely: (I) polyhydric alcohol, (II) unsaturated polybasic acid, (III) saturated polybasic acid and (IV) reactive monomer.

\section{Polyhydric alcohol}

Polyhydric alcohol component of the polyester structure has as important influence on the properties of the cross-linked copolymer as any of the acids used. The principal alcohols used in polyester synthesis are dihydric alcohols (glycols). Monohydric alcohols are sometimes used to determine the chain growth. Alcohols that contain more than two hydroxyl groups give branching in the polyester chain [2].

Diethylene glycol (IUPAC name 2,2'-Oxydi(ethan-1-ol)) is classed as dihydric alcohol [4]. DEG is a most common abbreviation for the diethylene glycol. DEG is produced by the partial hydrolysis of ethylene oxide and the resulting product is two ethylene glycol molecules joined by an ether bond [5].

Propylene glycol (IUPAC name: propane-1,2-diol) chemically is, also, classed as a diol, i.e. dihydric alcohol [4]. Propylene glycol (abbreviated PPG) is sometimes called $\alpha$ propylene glycol to distinguish it from the isomer propane1,3-diol ( $\beta$-propylene glycol). It gives non-crystallizing polyester resin completely compatible with styrene. This glycol is relatively cheap and thus is used in the bulk of all polyester resin produced today.

Neopentyl glycol (IUPAC name: 2,2-dimethylpropane-1,3diol) is dihydric alcohol containing two primary hydroxyl groups [4]. It is synthesized industrially by the aldol reaction of formaldehyde and isobutyraldehyde [6]. Sometimes it is blended with other glycols to improve the styrene compatibility of the resin.

The other dihydric alcohols are ethylene glycol, dipropylene glycol, dibromoneopentyl glycol, etc.

Pentaerythritol (IUPAC name 2,2-Bis (hydroxymethyl) propane-1,3-diol) is polyhydric alcohol with more than two hydroxyl groups [4]. The word pentaerythritol (abbreviated in a polyester resin terminology PET) is a linguistic blend of words penta- in reference to the number of carbon atoms and erythritol, which possesses 4 hydroxyl groups. This alcohol is used in the manufacture of polyester resins to introduce branching in the polyester chain.

The other polyhydric alcohols are glycerol, mannitol, etc.

Skeletal formulae of diethylene glycol (a), propylene glycol (b), neopentyl glycol (c) and pentaerythritol (d) are presented in Fig.5.<smiles>OCCOCCO</smiles>

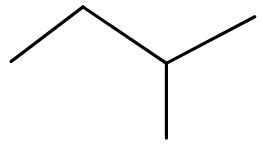

(a)

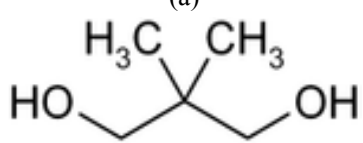

(c) (b)

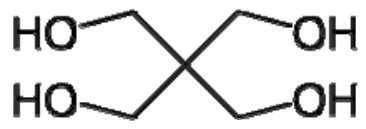

(d)
Figure 5. Skeletal formulae of diethylene glycol (a), propylene glycol (b), neopentyl glycol (c) and pentaerythritol (d)

\section{Unsaturated polybasic acids}

Unsaturated polybasic acid present in the polymer chain enables it to be cross-linked. The higher the proportion is present, the higher the degree of cross-linking. This component, also, affects the reactivity of resin. The higher the degree of unsaturation, the higher is the reactivity of the resin. The most commonly used unsaturated polybasic organic acid is maleic acid (melting point range from $132{ }^{\circ} \mathrm{C}$ to $140{ }^{\circ} \mathrm{C}$ ), generally used as the anhydride (melting point $60^{\circ} \mathrm{C}$ ) due to its lower melting point.

Maleic anhydride (IUPAC name Furan-2,5-dione) is the acid anhydride of maleic acid [4]. Maleic anhydride is produced by vapor-phase oxidation of $n$-butane and formula (skeletal) is presented in Fig.6.

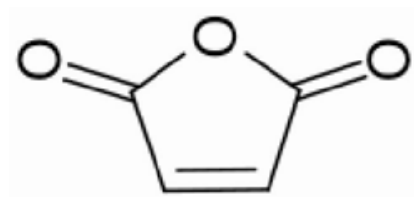

Figure 6. Skeletal formula of anhydride of maleic acid

Other unsaturated polybasic acids are chloromaleic acid, itaconic acid, citraconic acid, etc.

Reactivity and cured properties of a polyester resins can be modified by blending unsaturated polybasic acids with saturated polybasic acids.

\section{Saturated polybasic acids}

The term saturated is used to describe those dibasic acids or anhydrides which do not contain pendant double bonds, which react in the present of the catalyst. They may contain aromatic nucleus.

The anhydride of orthophthalic acid (IUPAC name 2Benzofuran-1,3-dione) was first suggested for use in polyester production [4]. This compound is often just called anhydride phthalic acid (abbreviated APA) because it is only isomer capable of forming an anhydride. Resins prepared with this anhydride are clear and have good compatibility with styrene. Since it is relatively cheap, easy available and since polyester resin prepared from it have good all-round properties, it has remained one of the most widely used anhydride.

The isophthalic acid (IUPAC name Benzene-1,3dicarboxylic acid) is the next most commonly used saturated acid, more expensive than APA, but it gives resins with higher molecular weight [4].

The other saturated acids/anhydrides are sebacic acid, tetrahydrophthalic anhydride, hexachloro-endo-methylenetetrahydrophthalic anhydride (known as HET or chlorendic anhydride), etc.

Skeletal formulae of anhydride of phthalic acid (a) and isophthalic acid (b) are presented in Fig.7. 


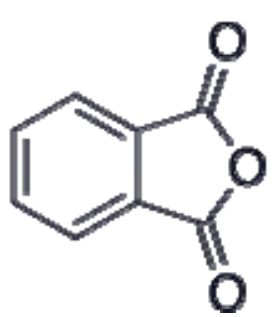

(a)

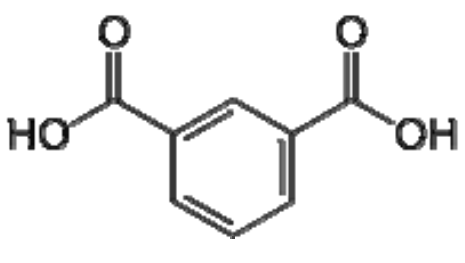

(b)
Figure 7. Skeletal formulae of anhydride of phthalic acid (a) and isophthalic acid (b)

\section{Monomer}

The monomer serves two purposes: (a) to act as solvent for a polyester resin to produce a liquid with a suitable handling viscosity and (b) to connect the polyester chains with double bonds to give fully cross-linked thermoset structure in copolymerization reaction.

Styrene (IUPAC name Ethenylbenzene) is characterized by low viscosity, low cost and ready availability [4]. The formula of the most frequently used monomer is presented in Fig.8.

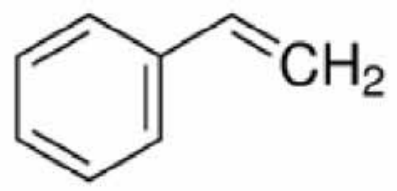

Figure 8. Skeletal formula of styrene

The other monomers are methyl methacrylate, dichlorostyrene and $\alpha$-methyl styrene.

Other, frequently, used components in fabrication of polyester resin-based products is catalysts, accelerators and inhibitors [3].

Catalyst or initiators for unsaturated a polyester resin systems consist of organic peroxides which yield highly reactive free radical species [8]. Cure of unsaturated a polyester resin takes place in polymerization process through unsaturated groups both in polyester chain and the monomer, initiated by free radical. The free radicals are provided by the peroxide as it decomposes and it is the rate at which these free radicals are produced which governs the gel and cure time of the resin. Organic peroxides can be divided into two broad classes: the true peroxides (as benzoyl peroxide) and the hydroperoxides (as cumene hydroperoxides). The most commonly encountered peroxide catalyst for room temperature cure is methyl ethyl ketone peroxide (abbreviated MEK peroxide), which is considered to be mixed peroxide. MEK peroxide is very explosive in its pure form and is therefore always supplied diluted in plasticizer, usually dimethyl phthalate or dibutyl phthalate.

Accelerators or promoters are materials that are used in conjunction with an organic peroxide catalyst to increase the rate at which peroxide breaks down into free radicals. Thus, they accelerate the cure of the polyester resin in controlled manner. They can be divided into two classes of materials metal compounds and tertiary amines. Metal compounds are usually metal salts or soaps in solutions of different plasticizers. Cobalt salts are frequently and manganese and vanadium accelerators occasionally used. Cobalt naphthenate (diluted into styrene) is mostly used because it is excellent accelerator for hydroperoxides and mixed peroxides.

Inhibitors are added to ensure adequate shelf-life of resin. Some inhibitors increase gel time and cure time as well as pot-life, whilst others just increase pot-life without influencing two mentioned technological parameters. Two inhibitors which are used to prolong pot-life are t-butyl catechol and di-t-butyl-p-cresol.

The curing of a polyester resin can be considered to take place in three stages [3]:

1. Gelation - where resin changes from a free-flowing liquid to a soft gel.

2. Hardening - where the resin cures from a soft gel to a hard material capable of being removing from the mold.

3. Post cure or maturing - where the resin achieves its full mechanical, physical and chemical properties. For room temperature cured systems, post curing or final curing may be carried out at elevated temperature. This process should always be carried out immediately after hardening stage while there is still sufficient residual peroxide to complete cure.

All resins absorb water to a greater or lesser extent. Water is, indeed, an important degradation factor for polyester-resin based polymeric products [8]. The absorption of a resin can be attributed to the moisture affinity of the highly polar functional group in the cured resin. In a polyester resin the higher molecular weight, the smaller will be the number of the hydrophilic end groups of the polyester ingredients [9].

Material characteristics are defined as measuring values, by which the shape or the measure of material capability to react to external influence are characterized. In accordance with the nature of external influences on a material, material characteristics can be classified into three groups: 1) mechanical characteristics, 2) physical characteristics and 3) chemical characteristics [10]. Mechanical properties refer to the material behavior under the influence of external mechanical forces. Physical properties refer to the material behavior under physical stress.

Water absorption content and quantity of water soluble matter are materials characteristics that reflect the quality changes of the all cured polyester resins during tested periods of time in mentioned liquids.

The effects of the water on the resin can be to cause plasticization process, swelling process and bond breaking process. It is stated that most of the changes, resulting from the expansion of the resin by water, occur in the vicinity of the surface of a polyester resin materials. Investigation of Barcol hardness belongs to so called shaped matter indention tests. Barcol hardness measures the resistance of the surface and nearby polyester resin material to penetration of sharp steel part under spring.

It can be supposed that some water pick-up effectively "anneals" polyester resin product. Impact resistance is measure of energy required to break a specimen and thus is property of the whole polyester resin based product. In other words, impact resistance is measure of the characteristic of total mass of the polyester resin system material.

Barcol hardness was chosen as an important parameter of the quality of a polyester resin system [11]. The same refers to impact resistance.

Eckstein, who examined over 70 dissimilar resin formulations, has concluded that water absorption may differ by a factor of 10 between various resin types and up to three for the same resin with a different curing agent [12].

Pritchard and Speake predicted that $3.6 \%$ water would be absorbed by the cast unreinforced PR after ten years - this is about the same as after three days at $100^{\circ} \mathrm{C}$, thus emphasizing the severity of boiling water test [13].

Belan, Bellenger, Mortaigne and Verdu tested propylene glycol/neopentyl glycol-maleate/isophthalate copolymers crosslinked by styrene at various temperatures between $30^{\circ} \mathrm{C}$ 
and $90{ }^{\circ} \mathrm{C}$. They concluded that water concentration increases with the temperature and that material expansion is induced by temperature [14].

For the purpose of this paper, impact resistance and Barcol hardness, as a mechanical characteristics, and water absorption content and quantity of water soluble matter, as physical properties, are chosen to follow the influence of a polyester resin ingredients and distillated and synthetic sea water at laboratory temperature and distillated water at boiling temperature to quality of four tested polyester resin based systems.

In this paper the influence of ingredients of a polyester resin and the influence of water of laboratory and elevated temperature on mechanical and physical characteristics of cured four different polyester systems are presented.

\section{Experimental part}

Next abbreviations of ingredients and monomer (for the purpose of this paper) will be used: diethylene glycol (DEG), propylene glycol (PPG), neopentyl glycol (NPG), pentaerythritol (PET), anhydride of phthalic acid (APA), isophthalic acid (IPA), anhydride of maleic acid (AMA) and styrene $(\mathrm{St})$.

Polyester resin system (abbreviated for the purpose of this paper - PR system) mark 1 consists of DEG/PPG/APA/AMK/St, PR system mark 2 of PET/PPG/APA/AMK/St, PR system mark 3 of PPG/IPA/AMK/St and PR system mark 4 of NPG/IPA/AMK/St.

Common properties of mentioned four PR system (visual appearance, viscosity, acid number and gel time) are presented in Table 1.

Table 1. Common properties of PR system mark1, PR system mark 2, PR system mark 3 and PR system mark 4

\begin{tabular}{||c|c|c|c|c||}
\hline Property & $\begin{array}{c}\text { PR system } \\
\text { mark 1 }\end{array}$ & $\begin{array}{c}\text { PR system } \\
\text { mark 2 }\end{array}$ & $\begin{array}{c}\text { PR system } \\
\text { mark 3 }\end{array}$ & $\begin{array}{c}\text { PR system } \\
\text { mark 4 }\end{array}$ \\
\hline \hline $\begin{array}{c}\text { Visual } \\
\text { appearance }\end{array}$ & $\begin{array}{c}\text { Transparent } \\
\text { light green }\end{array}$ & $\begin{array}{c}\text { Opaque milk } \\
\text { white }\end{array}$ & $\begin{array}{c}\text { Transparent } \\
\text { blue }\end{array}$ & $\begin{array}{c}\text { Transparent } \\
\text { light brown }\end{array}$ \\
\hline $\begin{array}{c}\text { Viscosity, Ford, } \\
\varnothing ~ 4 / 20^{\circ} \mathrm{C}, \mathrm{s}\end{array}$ & 140 & 175 & 134 & 230 \\
\hline Acid number & 37,5 & 28,0 & 16,0 & 20,6 \\
\hline Gel time*, min/s & $13 / 13$ & $9 / 30$ & $9 / 55$ & $10 / 25$ \\
\hline
\end{tabular}

* for determination of gel time were used $3 \% \mathrm{~m} / \mathrm{m}$ (based on the resin mass) of the solution of methyl ethyl ketone peroxide in dibutyl phthalate (concentration $50 \%$ ) as catalyst and $2 \% \mathrm{~m} / \mathrm{m}$ (based on the resin mass) of the solution of cobalt-naphtenate diluted into styrene (concentration of $1 \%$ ) as accelerator.

Using each of four mentioned PR system and the catalyst and an accelerator (applied for determination of gel time) a homogenized mixtures were produced and poured in metal mold dimensions $120 \mathrm{~mm}$ x $15 \mathrm{~mm}$ x $10 \mathrm{~mm}$. Homogenized mixtures were allowed to hardened for 24 hours at $23{ }^{\circ} \mathrm{C}$, afterwards the post curing procedure were applied ( 2 hours at $60{ }^{\circ} \mathrm{C}$ and $7 \times 24$ hours at $23^{\circ} \mathrm{C}$ ).

One part of specimen dimensions $120 \mathrm{~mm}$ x $15 \mathrm{~mm}$ x 10 $\mathrm{mm}$ is intended for determination of water absorption, watersoluble matter and impact resistance. Another part of hardened and post cured bars dimensions $120 \mathrm{~mm}$ x $15 \mathrm{~mm}$ x $10 \mathrm{~mm}$ of all four mentioned PR systems was used for production of specimen shape prism dimensions $25 \mathrm{~mm} \times 15$ $\mathrm{mm} \times 10 \mathrm{~mm}$ by machining. The later specimens are intended for determination of water absorption, water-soluble matter and Barcol hardness.

These specimens are used for determining mechanical characteristics (impact resistance and Barcol hardness) and physical properties (water absorption and water soluble matter) before, during and after exposure to the influence of distillated water and synthetic sea water at $23{ }^{\circ} \mathrm{C}$ and distillated water at boiling temperature $\left(100^{\circ} \mathrm{C}\right)$ [15].

Barcol hardness is very simple, fast, and useful test used to determine the degree of cure as a parameter of PR system quality. Generally, a well fabricated, well cured product will have a minimum 30 Barcol degrees [16].

Unsaturated PR system is the most important polymers for production of casting resins (no reinforced) and composite (reinforced) materials [17]. These materials are used in many different areas, including those in which the products are exposed to the influence of water [18].

Although it is difficult to predict on the basis of laboratory data alone how any specific polyester product will stand up on exposure to a particular environment and chemicals, results, obtained by testing specimens in conditions similar to those in which produced part will be used, can be very helpful.

Specimens of four PR systems were exposed to the influence of water. Specimens of dimensions $25 \mathrm{~mm} \times 15 \mathrm{~mm}$ x $10 \mathrm{~mm}$ and of dimensions $120 \mathrm{~mm}$ x $15 \mathrm{~mm}$ x $10 \mathrm{~mm}$ were exposed to the influence of distillated water and synthetic sea water at $23^{\circ} \mathrm{C}$ for a period of 30 days and distillated water at boiling temperature $\left(100{ }^{\circ} \mathrm{C}\right)$ for a period of 210 minutes.

\section{Results and analysis}

In accordance with the procedure described in experimental part of this paper, appropriate sets of specimens dimensions $120 \mathrm{~mm} \times 15 \mathrm{~mm} \times 10 \mathrm{~mm}$ and appropriate sets of specimens dimensions $25 \mathrm{~mm}$ x $15 \mathrm{~mm}$ x $10 \mathrm{~mm}$ of all four mentioned PR systems were produced.

Four sets of specimens dimensions $120 \mathrm{~mm}$ x $15 \mathrm{~mm}$ x 10 $\mathrm{mm}$ of all four mentioned PR systems were immersed in distillated water at $23{ }^{\circ} \mathrm{C}$ for a period of thirty days. During mentioned period, after certain periods, specimens were removed from the water, treated in accordance with appropriate standards, and water absorption, water soluble matter and impact resistance were tested.

Four sets of specimens of all four mentioned PR systems, which have above mentioned dimensions, were immersed in synthetic sea water at $23{ }^{\circ} \mathrm{C}$. This investigation lasted for a period of thirty days and after a while, specimens were removed from the mentioned liquid, treated in accordance with appropriate standards, and water absorption, water soluble matter and impact resistance were tested.

Two mentioned investigations were done, also, with appropriate number of specimens dimensions $25 \mathrm{~mm}$ x $15 \mathrm{~mm}$ x $10 \mathrm{~mm}$ of all four mentioned PR systems, i.e. four sets of this specimens were immersed in distillated water at $23^{\circ} \mathrm{C}$ for a period of thirty days and another four sets of specimens of same dimensions were immersed in synthetic sea water at $23{ }^{\circ} \mathrm{C}$ for the same period of time. During mentioned time, after certain periods, the specimens were removed from the appropriate water, treated in accordance with chosen standards, and water absorption, water soluble matter and Barcol hardness were tested.

Synthetic sea water consists of the following ingredients: $\mathrm{NaCl}(26,52 \mathrm{~g} / \mathrm{l}), \mathrm{MgSO}_{4}(3,30 \mathrm{~g} / \mathrm{l}), \mathrm{MgCl}_{2}(2,45 \mathrm{~g} / \mathrm{l}), \mathrm{CaCl}_{2}$ $(1,14 \mathrm{~g} / \mathrm{l}), \mathrm{KCl}(0,73 \mathrm{~g} / \mathrm{l}), \mathrm{NaHCO}_{3}(0,20 \mathrm{~g} / \mathrm{l})$ and $\mathrm{NaBr}(0,08$ $\mathrm{g} / \mathrm{l})$ [19].

Specimens of dimensions $25 \mathrm{~mm} \times 15 \mathrm{~mm}$ x $10 \mathrm{~mm}$ of all four mentioned PR systems were exposed to the influence of boiling water for a period of 210 minutes and after every thirty minutes, specimens were removed, treated as it is defined in adequate standards, and water absorption, water 
soluble matter and Barcol hardness were tested.

Set of specimens dimensions $120 \mathrm{~mm} \times 15 \mathrm{~mm} \times 10 \mathrm{~mm}$ of all four mentioned PR systems were immersed in the boiling water for a period of 210 minutes. During this period, after every thirty minutes, specimens were removed from the boiling water, treated as it is defined in appropriate standards, and water absorption, water soluble matter and impact resistance were tested.

Water absorption and water soluble matter were tested in accordance with ISO 62 standard [20].

Impact resistance was determined following the instructions in ISO 179 standard [21].

Testing Barcol hardness was done in a line with ASTM D 2583-13a standard [22].

Viscosity, acid number and gel time were tested in accordance with ASTM D 1200, ISO 2114 and ISO 2535 standards, respectively [23, 24, 25].

Testing results with distillated and synthetic sea water and analysis

One specimen dimensions $25 \mathrm{~mm}$ x $15 \mathrm{~mm}$ x $10 \mathrm{~mm}$ (abbreviated for a table presentation in this paper-H.S.hardness specimen) and one specimen dimensions $120 \mathrm{~mm} \mathrm{x}$ $15 \mathrm{~mm} \times 10 \mathrm{~mm}$ (shortened for a table presentation in this paper -I.R.S. - impact resistance specimen) of each of four polyester resin systems were immersed in distillated water and synthetic sea water at $23^{\circ} \mathrm{C}$ for a period of thirty days. Data recorded during this testing and calculated corresponding water absorption (single value) and water soluble matter (single value) are presented in Table 2.

Table 2. Data recorded during testing one H.S. and one I.R.S. of each of four PR systems in distillated and synthetic sea water at $23{ }^{\circ} \mathrm{C}$ for a period of thirty days and corresponding water absorption and water soluble matter

\begin{tabular}{|c|c|c|c|c|c|c|c|c|}
\hline \multirow{3}{*}{$\begin{array}{c}\text { Specimen } \\
\text { mark }\end{array}$} & \multicolumn{8}{|c|}{ PR system mark } \\
\hline & \multicolumn{2}{|c|}{ Mark 1} & \multicolumn{2}{|c|}{ Mark 2} & \multicolumn{2}{|c|}{ Mark 3} & \multicolumn{2}{|c|}{ Mark 4} \\
\hline & H.S. & I.R.S. & H.S. & I.R.S. & H.S. & I.R.S. & H.S. & I.R.S. \\
\hline$m_{1}(\mathrm{~g})$ & 5.9625 & 18.7235 & 6.1475 & 18.2235 & 7.0025 & 18.8637 & 6,3825 & 19,0206 \\
\hline$m_{2,5, \text { d.w. }}(\mathrm{g})$ & 5.9748 & 18.7538 & 6.1568 & 18.2514 & 700117 & 18.8891 & 6,3890 & 19,0390 \\
\hline$C_{\text {a.w. }}(\%)$ & 0.162 & 0.162 & 0.151 & 0.153 & 0.132 & 0.135 & 0,102 & 0,097 \\
\hline$m_{3,5, \text { d.w.,c.m. }(\mathrm{g})}$ & 5.9561 & 18.6980 & 6.1400 & 18.2040 & 6.9930 & 18.8419 & 6,3770 & 1900066 \\
\hline$C_{\text {w.s.m. }}(\%)$ & 0.314 & 0.298 & 0.272 & 0.253 & 0.267 & 0.250 & 0,188 & 0,179 \\
\hline$m_{1}(\mathrm{~g})$ & 5.8635 & 18.2835 & 6.3628 & 18.4435 & 6.4758 & 18.4588 & 6.1898 & 19.2531 \\
\hline$m_{2,5, \text { s.s.w. }}(\mathrm{g})$ & 5.8728 & 18.3120 & 6.3708 & 18.4663 & 6.4832 & 18.4809 & 6.1959 & 19.2689 \\
\hline$C_{\text {a.w. }}(\%)$ & 0.159 & 0.156 & 0.126 & 0.124 & 0.114 & 0.120 & 0.099 & 0.082 \\
\hline$m_{3,5, \text { s.s.w.c.m. }}(\mathrm{g})$ & 5.8545 & 18.2575 & 6.3541 & 18.4178 & 6.4662 & 18.4368 & 6.1844 & 19.2342 \\
\hline$C_{\text {w.s.m. }}(\%)$ & 0.311 & 0.298 & 0.263 & 0.263 & 0.263 & 0.239 & 0.186 & 0.180 \\
\hline$m_{1}(\mathrm{~g})$ & 5.6988 & 19.8878 & 7.9452 & 21.7649 & 5.9209 & 19.6774 & 6.4666 & 20.3623 \\
\hline$m_{, 2,10, \text { d.w. }}(\mathrm{g})$ & 5.7145 & 19.9427 & 7.9626 & 21.8217 & 5.9342 & 19.7232 & 6.4793 & 20.3963 \\
\hline$C_{\text {a.w. }}(\%)$ & 0.276 & 0.276 & 0.219 & 0.261 & 0.225 & 0.233 & 0.196 & 0.176 \\
\hline$m_{3,10, \text { d.w.,c.m. }}(\mathrm{g})$ & 5.6840 & 19.8381 & 7.9224 & 21.7144 & 5.9061 & 19.6346 & 6.4570 & 20.3222 \\
\hline$C_{\text {w.s.m. }}(\%)$ & 0.534 & 0.526 & 0.506 & 0.493 & 0.475 & 0.450 & 0.342 & 0.364 \\
\hline$m_{1}(\mathrm{~g})$ & 6.9865 & 20.5219 & 5.6359 & 20.0696 & 5.7030 & 19.1137 & 6.8944 & 18.5918 \\
\hline$m_{2,10, \text { s.s.w. }}(\mathrm{g})$ & 7.0044 & 20.5715 & 5.6714 & 20.1139 & 5.7146 & 19.1554 & 6.9050 & 18.6191 \\
\hline$C_{\text {a.w. }}(\%)$ & 0.256 & 0.242 & 0.214 & 0.221 & 0.203 & 0.218 & 0.154 & 0.147 \\
\hline$m_{3,10, \text { s.s.w.c.m.m. }}(\mathrm{g})$ & 6.9682 & 20.3269 & 5.6450 & 20.0165 & 5.6895 & 19.0720 & 6.8823 & 18.5616 \\
\hline$C_{\text {w.s.m. }}(\%)$ & 0.518 & 0.484 & 0.466 & 0.485 & 0.440 & 0.436 & 0.329 & 0.309 \\
\hline$m_{1}(\mathrm{~g})$ & 6.9086 & 20.0454 & 5.0245 & 21.9075 & 6.8003 & 20.3595 & 5.6742 & 18.9792 \\
\hline$m_{2,20, \text { d.w. }}(\mathrm{g})$ & 7.0079 & 20.1276 & 5.0424 & 21.9914 & 6.8209 & 20.4256 & 5.6900 & 190.0262 \\
\hline$C_{\text {a.w. }}(\%)$ & 0.392 & 0.410 & 0.356 & 0.383 & 0.313 & 0.325 & 0.278 & 0.266 \\
\hline$m_{3,20, \text { d.w.c.m. }}(\mathrm{g})$ & 6.9642 & 20.0009 & 5.0115 & 21.8593 & 6.7860 & 20.3065 & 5.666. & 18.9501 \\
\hline$C_{\text {w.s.m. }}(\%)$ & 0.626 & 0.632 & 0.615 & 0.603 & 0.513 & 0.585 & 0.418 & 0.401 \\
\hline$m_{1}(\mathrm{~g})$ & 7.2701 & 19.8593 & 7.9994 & 20.2571 & 6.6924 & 21.0801 & 7.5182 & 19.2717 \\
\hline$m_{2,20, \text { s.s.w. }}(\mathrm{g})$ & 7.2962 & 19.9335 & 8.0246 & 20.3223 & 6.7114 & 21.1416 & 7.5376 & 19.3170 \\
\hline$C_{\text {a.w. }}(\%)$ & 0.359 & 0.374 & 0.315 & 0.322 & 0.284 & 0.292 & 0.258 & 0.235 \\
\hline$m_{3,20, \text { s.s.w.,c.m. }}(\mathrm{g})$ & 7.2531 & 19.8143 & 7.9805 & 20.2080 & 6.6782 & 21.0376 & 7.5079 & 19.2406 \\
\hline$C_{\text {w.s.m. }}(\%)$ & 0.593 & 0.598 & 0.551 & 0.564 & 0.496 & 0.493 & 0.395 & 0.396 \\
\hline$m_{1}(\mathrm{~g})$ & 7.6981 & 20.4324 & 7.2316 & 20.8083 & 8.2794 & 21.4242 & 7.7536 & 20.3905 \\
\hline$m_{, 2,30, \text { d.w. }}(\mathrm{g})$ & 7.7352 & 20.5628 & 7.2658 & 20.9051 & 8.3106 & 21.5771 & 7.7793 & 20.4604 \\
\hline$C_{\text {a.w }}(\%)$ & 0.482 & 0.491 & 0.473 & 0.465 & 0.377 & 0.388 & 0.331 & 0.343 \\
\hline$m_{3,30, \text { d.w.,c.m. }}(\mathrm{g})$ & 7.6801 & 20.4193 & 7.2220 & 20.7802 & 8.2680 & 21.3809 & 7.7483 & 20.3702 \\
\hline$C_{\text {w.s.m. }}(\%)$ & 0.715 & 0.701 & 0.606 & 0.600 & 0.515 & 0.585 & 0.400 & 0.442 \\
\hline$m_{1}(\mathrm{~g})$ & 7.9748 & 21.6092 & 7.3973 & 20.0921 & 7.4873 & 20.6287 & 5.5624 & 19.6692 \\
\hline$m_{2,30, \text { s.s.w. }}(\mathrm{g})$ & 8.0104 & 21.7088 & 7.4276 & 20.1807 & 7.5114 & 20.7006 & 5.5785 & 19.7270 \\
\hline$C_{\text {a.w. }}(\%)$ & 0.447 & 0.461 & 0.410 & 0.441 & 0.322 & 0.355 & 0.289 & 0.294 \\
\hline$m_{3,30, \text { s.s.w.,c.m. }}(\mathrm{g})$ & 7.9622 & 21.5672 & 7.3846 & 20.0609 & 7.4718 & 20.5883 & 5.5530 & 19.6448 \\
\hline$C_{\text {w.s.m. }}(\%)$ & 0.604 & 0.655 & 0.582 & 0.596 & 0.529 & 0.544 & 0.458 & 0.418 \\
\hline
\end{tabular}


Single values $\left(x_{\mathrm{i}}\right)$ and arithmetic mean value $(\bar{x} \pm \delta)$ for water absorption of specimens marks H.S. and I.R.S. of all four mentioned PR systems in distillated water at $23{ }^{\circ} \mathrm{C}$ for the periods of 5, 10, 20 and 30 days are presented in Table 3.

Arithmetic mean value is based on three single values. This refers to all arithmetic mean values in this paper.

Table 3. Water absorption of specimens marks H.S. and I.R.S. of all four mentioned PR systems in distillated water at $23{ }^{\circ} \mathrm{C}$ for the periods of $5,10,20$ and 30 days

\begin{tabular}{|c|c|c|c|c|c|c|c|c|c|}
\hline \multirow{3}{*}{\multicolumn{2}{|c|}{ PR system mark }} & \multicolumn{8}{|c|}{ Exposure time (day) } \\
\hline & & \multicolumn{2}{|c|}{5} & \multicolumn{2}{|c|}{10} & \multicolumn{2}{|c|}{20} & \multicolumn{2}{|c|}{30} \\
\hline & & H.S. & I.R.S. & H.S. & I.R.S. & H.S. & I.R.S. & H.S. & I.R.S. \\
\hline \multirow{4}{*}{ Mark 1} & \multirow{3}{*}{$\begin{array}{l}\text { (xi) } \\
(\%)\end{array}$} & 0.181 & 0.162 & 0.276 & 0.276 & 0.390 & 0.418 & 0.502 & 0.489 \\
\hline & & 0162 & 0.177 & 0.289 & 0.289 & 0.436 & 0.410 & 0.499 & 0.491 \\
\hline & & 0.179 & 0.165 & 0.270 & 0.270 & 0.392 & 0.393 & 0.482 & 0.490 \\
\hline & $\begin{array}{c}(\bar{x} \pm \delta) \\
(\%)\end{array}$ & $\begin{array}{c}0.174 \pm \\
0.01\end{array}$ & $\begin{array}{c}0.168 \pm \\
0.008\end{array}$ & $\begin{array}{c}0.278 \pm \\
0.01\end{array}$ & $\begin{array}{c}0.278 \pm \\
0.01\end{array}$ & $\begin{array}{c}0.406 \pm \\
0.01\end{array}$ & $\begin{array}{c}0.407 \pm \\
0.002\end{array}$ & $\begin{array}{c}0.494 \pm \\
0.01\end{array}$ & $\begin{array}{c}0.490 \pm \\
0.001\end{array}$ \\
\hline \multirow{5}{*}{ Mark 2} & \multirow{3}{*}{$\begin{array}{l}\text { (xi) } \\
(\%)\end{array}$} & 0.157 & 0.153 & 0.231 & 0.231 & 0.356 & 0.383 & 0.478 & 0.449 \\
\hline & & 0.151 & 0.140 & 0.341 & 0.341 & 0.451 & 0.384 & 0.473 & 0.465 \\
\hline & & 0.147 & 0.154 & 0.219 & 0.219 & 0.351 & 0.383 & 0.452 & 0.470 \\
\hline & $(\bar{x} \pm \delta)$ & $0.152 \pm$ & $0.149 \pm$ & $0.264 \pm$ & $0.264 \pm$ & $0.386 \pm$ & $0.383 \pm$ & $0.468 \pm$ & $0.461 \pm$ \\
\hline & $(\%)$ & 0.005 & 0.008 & 0.07 & 0.07 & 0.03 & 0.001 & 0.01 & 0.001 \\
\hline \multirow{5}{*}{ Mark 3} & \multirow{3}{*}{$\begin{array}{l}\text { (xi) } \\
\text { (\%) }\end{array}$} & 0.129 & 0.135 & 0.225 & 0.225 & 0.370 & 0.325 & 0.422 & 0.383 \\
\hline & & 0.141 & 0.149 & 0.253 & 0.253 & 0.303 & 0.320 & 0.377 & 0.388 \\
\hline & & 0.132 & 0.136 & 0.208 & 0.208 & 0.299 & 0.329 & 0.387 & 0.402 \\
\hline & $(\bar{x} \pm \delta)$ & $0.134 \pm$ & $0.140 \pm$ & $0.229 \pm$ & $0.229 \pm$ & $0.324 \pm$ & $0.325 \pm$ & $0.395 \pm$ & $0.391 \pm$ \\
\hline & $(\%)$ & 0.006 & 0.008 & 0.02 & 0.02 & 0.01 & 0.005 & 0.02 & 0.01 \\
\hline \multirow{5}{*}{ Mark 4} & \multirow{3}{*}{$\begin{array}{l}\text { (xi) } \\
(\%)\end{array}$} & 0.112 & 0.093 & 0.174 & 0.172 & 0.278 & 0.271 & 0.358 & 0346 \\
\hline & & 0.101 & 0.107 & 0.196 & 0.167 & 0.281 & 0.264 & 0.367 & 0.338 \\
\hline & & 0.102 & 0.097 & 0.166 & 0.166 & 0.273 & 0.263 & 0.331 & 0.343 \\
\hline & $(\bar{x} \pm \delta)$ & $0.105 \pm$ & $0.099 \pm$ & $0.179 \pm$ & $0.168 \pm$ & $0.277 \pm$ & $0.266 \pm$ & $0.352 \pm$ & $0.342 \pm$ \\
\hline & (\%) & 0.006 & 0.007 & 0.02 & 0.003 & 0.004 & 0.004 & 0.02 & 0.004 \\
\hline
\end{tabular}

Dependence of arithmetic mean values of water absorption of exposure time [specimen H.S. (a) and specimen I.R.S. (b)] for

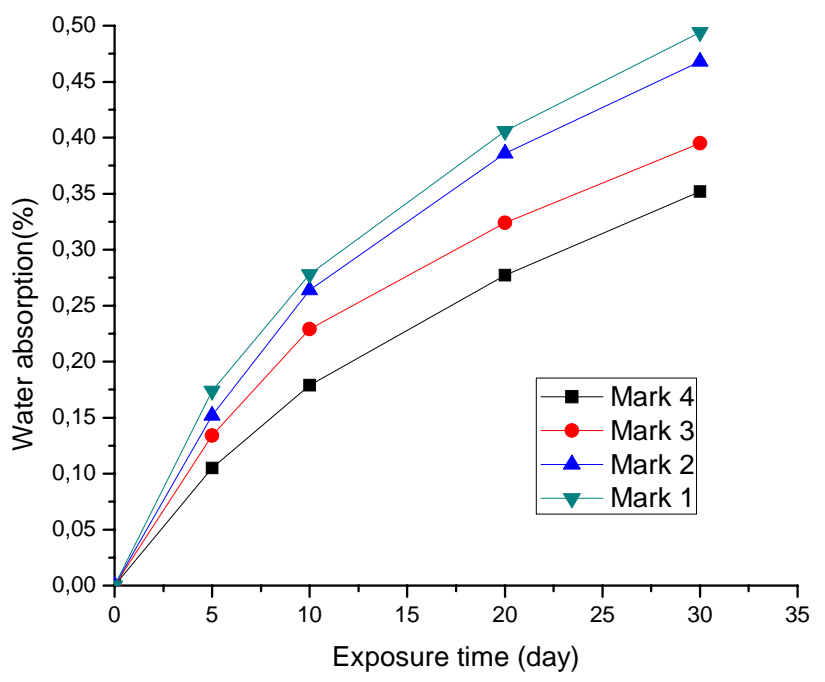

(a) all four PR systems in distillated water at $23{ }^{\circ} \mathrm{C}$ for a period of thirty days are presented in Fig.9.

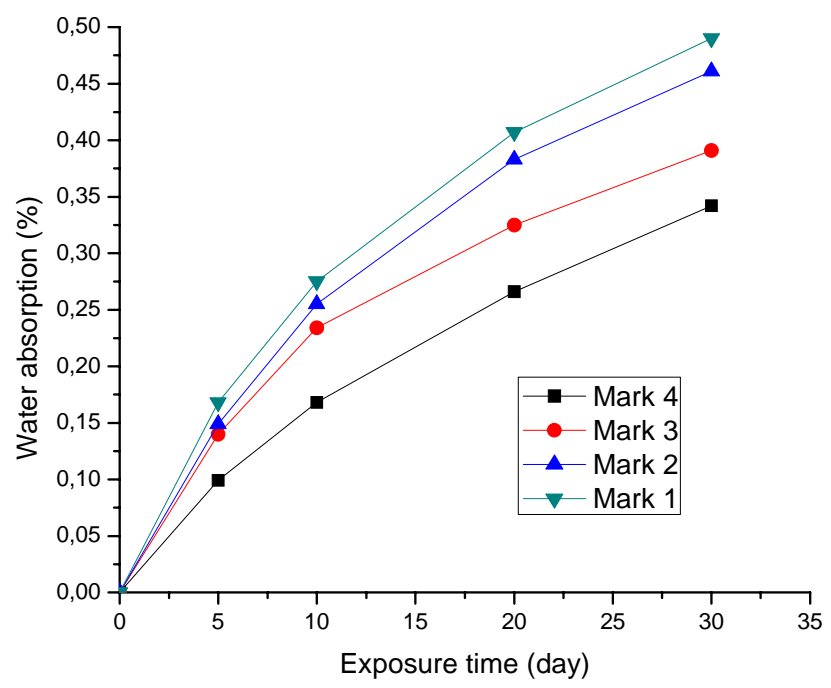

(b)

Figure 9. Dependence of water absorption of exposure time [specimen H.S. (a) and specimen I.R.S. (b)] for all four PR systems in distillated water at $23{ }^{\circ} \mathrm{C}$ for a period of thirty days

Data [single values $\left(x_{i}\right)$ and arithmetic mean value $\left.(\bar{x} \pm \delta)\right]$ for water absorption of specimens marks H.S. and I.R.S. of all four mentioned PR systems in synthetic sea water at $23^{\circ} \mathrm{C}$ for a period of thirty days is presented in Table 4 . 
Table 4. Water absorption of specimens marks H.S. and I.R.S. of all four mentioned PR systems in synthetic sea water at $23{ }^{\circ} \mathrm{C}$ for a period of thirty days

\begin{tabular}{|c|c|c|c|c|c|c|c|c|c|}
\hline \multirow{3}{*}{\multicolumn{2}{|c|}{ PR system mark }} & \multicolumn{8}{|c|}{ Exposure time (day) } \\
\hline & & \multicolumn{2}{|c|}{5} & \multicolumn{2}{|c|}{10} & \multicolumn{2}{|c|}{20} & \multicolumn{2}{|c|}{30} \\
\hline & & H.S. & I.R.S. & H.S. & I.R.S. & H.S. & I.R.S. & H.S. & I.R.S. \\
\hline \multirow[t]{2}{*}{ Mark 1} & $\begin{array}{l}\left(\mathrm{x}_{\mathrm{i}}\right) \\
(\%)\end{array}$ & $\begin{array}{l}0.166 \\
0.159 \\
0.158\end{array}$ & $\begin{array}{l}0.164 \\
0.151 \\
0.156\end{array}$ & $\begin{array}{l}0.267 \\
0.252 \\
0.256 \\
\end{array}$ & $\begin{array}{l}0.242 \\
0.271 \\
0.242 \\
\end{array}$ & $\begin{array}{l}0.394 \\
0.358 \\
0.359 \\
\end{array}$ & $\begin{array}{l}0.374 \\
0.379 \\
0.372 \\
\end{array}$ & $\begin{array}{l}0.481 \\
0.426 \\
0.447 \\
\end{array}$ & $\begin{array}{l}0.436 \\
0.461 \\
0.476\end{array}$ \\
\hline & $\begin{array}{c}(\bar{x} \pm \delta) \\
(\%)\end{array}$ & $\begin{array}{c}0.161 \pm \\
0.004\end{array}$ & $\begin{array}{c}0.157 \pm \\
0.006\end{array}$ & $\begin{array}{c}0.258 \pm \\
0.01\end{array}$ & $\begin{array}{c}0.252 \pm \\
0.02\end{array}$ & $\begin{array}{c}0.370 \pm \\
0.01\end{array}$ & $\begin{array}{c}0.375 \pm \\
0.007\end{array}$ & $\begin{array}{c}0.451 \pm \\
0.03\end{array}$ & $\begin{array}{c}0.458 \pm \\
0.02\end{array}$ \\
\hline \multirow[t]{2}{*}{ Mark 2} & $\begin{array}{l}\left(\mathrm{x}_{\mathrm{i}}\right) \\
(\%)\end{array}$ & $\begin{array}{l}0.118 \\
0.126 \\
0.116\end{array}$ & $\begin{array}{l}0.124 \\
0.116 \\
0.126 \\
\end{array}$ & $\begin{array}{l}0.214 \\
0.218 \\
0.216 \\
\end{array}$ & $\begin{array}{l}0.209 \\
0.221 \\
0.217 \\
\end{array}$ & $\begin{array}{l}0.378 \\
0.297 \\
0.315 \\
\end{array}$ & $\begin{array}{l}0.316 \\
0.322 \\
0.328 \\
\end{array}$ & $\begin{array}{l}0.410 \\
0.411 \\
0.449 \\
\end{array}$ & $\begin{array}{l}0.430 \\
0.404 \\
0.441 \\
\end{array}$ \\
\hline & $\begin{array}{c}(\bar{x} \pm \delta) \\
(\%)\end{array}$ & $\begin{array}{c}0.120 \pm \\
0.005\end{array}$ & $\begin{array}{c}0.122 \pm \\
0.005\end{array}$ & $\begin{array}{c}0.216 \pm \\
0.002\end{array}$ & $\begin{array}{c}0.213 \pm \\
0.01\end{array}$ & $\begin{array}{c}0.330 \pm \\
0.01\end{array}$ & $\begin{array}{c}0.322 \pm \\
0.06\end{array}$ & $\begin{array}{c}0.423 \pm \\
0.02\end{array}$ & $\begin{array}{c}0.425 \pm \\
0.002\end{array}$ \\
\hline \multirow[t]{2}{*}{ Mark 3} & $\begin{array}{l}\left(\mathrm{x}_{\mathrm{i}}\right) \\
(\%)\end{array}$ & $\begin{array}{l}0.116 \\
0.127 \\
0.114 \\
\end{array}$ & $\begin{array}{l}0.127 \\
0.116 \\
0.120 \\
\end{array}$ & $\begin{array}{l}0.203 \\
0.236 \\
0.202 \\
\end{array}$ & $\begin{array}{l}0.213 \\
0.223 \\
0.218 \\
\end{array}$ & $\begin{array}{l}0.316 \\
0.284 \\
0.275 \\
\end{array}$ & $\begin{array}{l}0.292 \\
0.290 \\
0.286 \\
\end{array}$ & $\begin{array}{l}0.378 \\
0.322 \\
0.355 \\
\end{array}$ & $\begin{array}{l}0.337 \\
0.347 \\
0.355 \\
\end{array}$ \\
\hline & $\begin{array}{c}(\bar{x} \pm \delta) \\
(\%)\end{array}$ & $\begin{array}{c}0.119 \pm \\
0.007\end{array}$ & $\begin{array}{c}0.121 \pm \\
0.005\end{array}$ & $\begin{array}{c}0.214 \pm \\
0.02\end{array}$ & $\begin{array}{c}0.218 \pm \\
0.005\end{array}$ & $\begin{array}{c}0.292 \pm \\
0.01\end{array}$ & $\begin{array}{c}0.288 \pm \\
0.005\end{array}$ & $\begin{array}{c}0.352 \pm \\
0.03\end{array}$ & $\begin{array}{c}0.346 \pm \\
0.009\end{array}$ \\
\hline \multirow[t]{2}{*}{ Mark 4} & $\begin{array}{l}\left(\mathrm{x}_{\mathrm{i}}\right) \\
(\%)\end{array}$ & $\begin{array}{l}0.090 \\
0.093 \\
0.099 \\
\end{array}$ & $\begin{array}{l}0.082 \\
0.099 \\
0.093 \\
\end{array}$ & $\begin{array}{l}0.164 \\
0.154 \\
0.144 \\
\end{array}$ & $\begin{array}{l}0.147 \\
0.150 \\
0.140 \\
\end{array}$ & $\begin{array}{l}0.267 \\
0.202 \\
0.258 \\
\end{array}$ & $\begin{array}{c}0.237 \\
0.2400 .235\end{array}$ & $\begin{array}{l}0.289 \\
0.305 \\
0.311 \\
\end{array}$ & $\begin{array}{l}0.312 \\
0.294 \\
0.284 \\
\end{array}$ \\
\hline & $\begin{array}{c}(\bar{x} \pm \delta) \\
(\%)\end{array}$ & $\begin{array}{c}0.094 \pm \\
0.05\end{array}$ & $\begin{array}{c}0.089 \pm \\
0.06\end{array}$ & $\begin{array}{c}0.154 \pm \\
0.01\end{array}$ & $\begin{array}{c}0.146 \pm \\
0.005\end{array}$ & $\begin{array}{c}0.242 \pm \\
0.04\end{array}$ & $\begin{array}{c}0.238 \pm \\
0.004\end{array}$ & $\begin{array}{c}0.302 \pm \\
0.01\end{array}$ & $\begin{array}{c}0.298 \pm \\
0.01\end{array}$ \\
\hline
\end{tabular}

Dependence of arithmetic mean values of water absorption of exposure time [specimen H.S. (a) and specimen I.R.S. (b)]

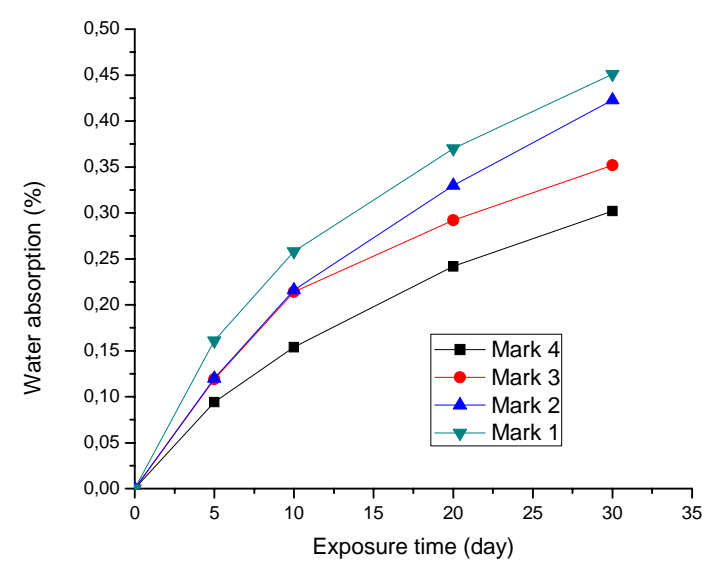

(a) for all four PR systems in synthetic sea water at $23{ }^{\circ} \mathrm{C}$ for a period of thirty days are presented in Fig. 10.

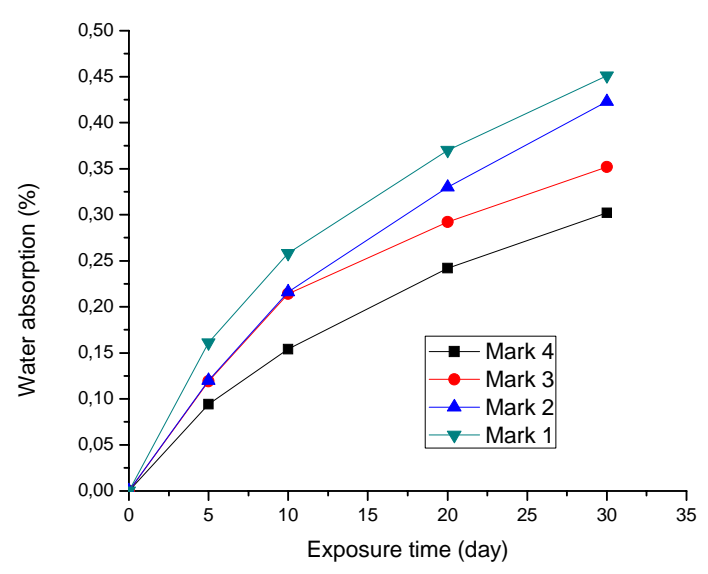

(b)

Figure 10. Dependence of water absorption of exposure time [specimen H.S. (a) and specimen I.R.S. (b)] for all four PR systems in synthetic sea water at $23{ }^{\circ} \mathrm{C}$ for a period of thirty days

Single values $\left(\mathrm{x}_{\mathrm{i}}\right)$ and arithmetic mean value $(\bar{x} \pm \delta)$ for water soluble matter of specimens marks H.S. and I.R.S. of all

four mentioned PR systems in distillated water at $23{ }^{\circ} \mathrm{C}$ for a period of thirty days are presented in Table 5.

Table 5. Water soluble matter of specimens marks H.S. and I.R.S. of all four mentioned PR systems in distillated water at $23{ }^{\circ} \mathrm{C}$ for a period of thirty days

\begin{tabular}{|c|c|c|c|c|c|c|c|c|c|}
\hline \multirow{3}{*}{\multicolumn{2}{|c|}{ PR system mark }} & \multicolumn{8}{|c|}{ Exposure time (day) } \\
\hline & & \multicolumn{2}{|c|}{5} & \multicolumn{2}{|c|}{10} & \multicolumn{2}{|c|}{20} & \multicolumn{2}{|c|}{30} \\
\hline & & H.S. & I.R.S. & H.S. & I.R.S. & H.S. & I.R.S. & H.S. & I.R.S. \\
\hline \multirow{5}{*}{ Mark 1} & \multirow{3}{*}{$\begin{array}{c}(\bar{x} \pm \delta) \\
(\%)\end{array}$} & 0.326 & 0.298 & 0.534 & 0.538 & 0.663 & 0.636 & 0.668 & 0.700 \\
\hline & & 0.314 & 0.312 & 0.539 & 0.526 & 0.585 & 0.632 & 0.705 & 0.701 \\
\hline & & 0.307 & 0.296 & 0.567 & 0.625 & 0.626 & 0.625 & 0.715 & 0.711 \\
\hline & $\left(\mathrm{x}_{\mathrm{i}}\right)$ & $0.315 \pm$ & $0.302 \pm$ & $0.542 \pm$ & $0.527 \pm$ & $0.625 \pm$ & $0.631 \pm$ & $0.696 \pm$ & $0.705 \pm$ \\
\hline & $(\%)$ & 0.009 & 0.008 & 0.02 & 0.005 & 0.04 & 0.006 & 0.02 & 0.008 \\
\hline \multirow{5}{*}{ Mark 2} & \multirow{3}{*}{$\begin{array}{c}(\bar{x} \pm \delta) \\
(\%)\end{array}$} & 0.280 & 0.253 & 0.516 & 0.495 & 0.615 & 0.604 & 0.626 & 0.609 \\
\hline & & 0.272 & 0.262 & 0.502 & 0.493 & 0.626 & 0.603 & 0.606 & 0.600 \\
\hline & & 0.267 & 265 & 0.506 & 0.482 & 0.579 & 0.590 & 0.6085 & 0.606 \\
\hline & $\left(\mathrm{x}_{\mathrm{i}}\right)$ & $0.273 \pm$ & $0.260 \pm$ & $0.580 \pm$ & $0.490 \pm$ & $0.607 \pm$ & $0.599 \pm$ & $0.613 \pm$ & $0.605 \pm$ \\
\hline & $(\%)$ & 0.006 & 0.006 & 0.07 & 0.007 & 0.02 & 0.008 & 0.01 & 0.005 \\
\hline
\end{tabular}




\begin{tabular}{|c|c|c|c|c|c|c|c|c|c|}
\hline \multirow{4}{*}{ Mark 3 } & $(\bar{x} \pm \delta)$ & 0.265 & 0.250 & 0.475 & 0.460 & 0.592 & 0.518 & 0.715 & 0.585 \\
& $(\%)$ & 0.251 & 0.258 & 0.485 & 0.450 & 0.513 & 0.520 & 0.524 & 0.588 \\
& $\left(\mathrm{x}_{\mathrm{i}}\right)$ & $0.261 \pm$ & $0.251 \pm$ & $0.461 \pm$ & $0.451 \pm$ & $0.534 \pm$ & $0.522 \pm$ & $0.584 \pm$ & $0.579 \pm$ \\
& $(\%)$ & 0.009 & 0.006 & 0.04 & 0.008 & 0.01 & 0.005 & 0.1 & 0.01 \\
\hline \multirow{3}{*}{ Mark 4 } & $(\bar{x} \pm \delta)$ & 0.186 & 0.193 & 0.451 & 0.350 & 0.418 & 0.407 & 0.421 & 0.432 \\
& $(\%)$ & 0.199 & 0.180 & 0.342 & 0.364 & 0.407 & 0.401 & 0.472 & 0.440 \\
& $(\bar{x} \pm \delta)$ & $0.191 \pm$ & $0.184 \pm$ & $0.372 \pm$ & $0.390 \pm$ & $0.415 \pm$ & $0.401 \pm$ & $0.431 \pm$ & $0.438 \pm$ \\
& $(\%)$ & 0.007 & 0.008 & 0.06 & 0.008 & 0.006 & 0.006 & 0.05 & 0.005 \\
\hline
\end{tabular}

Dependence of arithmetic mean values of water soluble matter of exposure time [specimen H.S. (a) and specimen

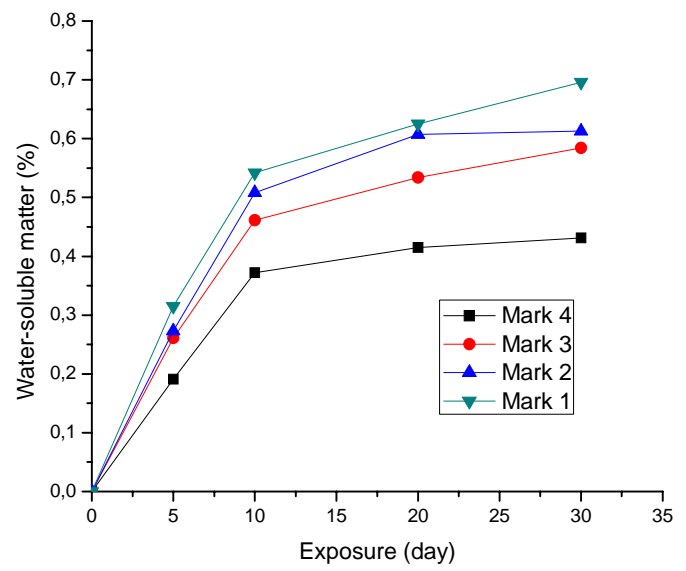

(a)
I.R.S. (b)] for all four PR systems in distillated water at $23{ }^{\circ} \mathrm{C}$ for a period of thirty days is presented in Figure 11.

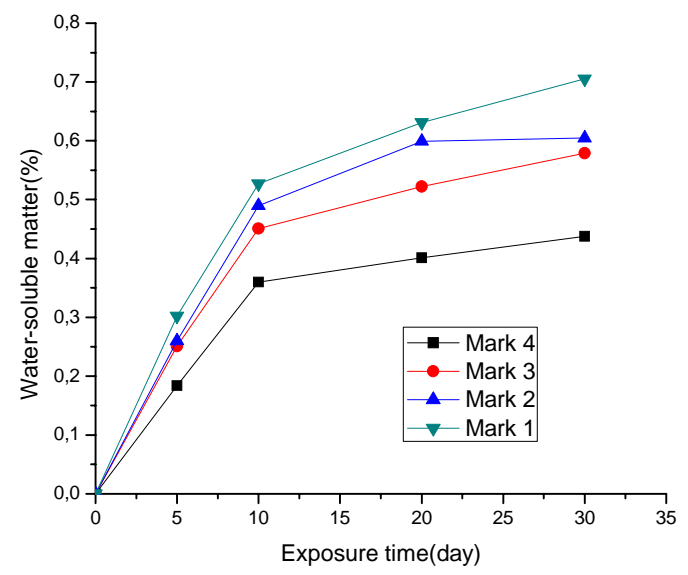

(b)

Figure 11. Dependence of water soluble matter of exposure time [specimen H.S. (a) and specimen I.R.S. (b)] for all four PR systems in distillated water at $23{ }^{\circ} \mathrm{C}$ for a period of thirty days

Data [single values $\left(x_{i}\right)$ and arithmetic mean value $\left.(\bar{x} \pm \delta)\right]$ for water soluble matter of specimens marks H.S. and I.R.S. of all four mentioned PR systems in synthetic sea water at $23{ }^{\circ} \mathrm{C}$ for a period of thirty days are presented in Table 6 .

Table 6. Water soluble matter of specimens marks H.S. and I.R.S. of all four mentioned PR systems in synthetic sea water at $23{ }^{\circ} \mathrm{C}$ for a period of thirty days

\begin{tabular}{|c|c|c|c|c|c|c|c|c|c|}
\hline \multirow{3}{*}{\multicolumn{2}{|c|}{ PR system mark }} & \multicolumn{8}{|c|}{ Exposure time (day) } \\
\hline & & \multicolumn{2}{|c|}{5} & \multicolumn{2}{|c|}{10} & \multicolumn{2}{|c|}{20} & \multicolumn{2}{|c|}{30} \\
\hline & & H.S. & I.R.S. & H.S. & I.R.S. & H.S. & I.R.S. & H.S. & I.R.S. \\
\hline \multirow{4}{*}{ Mark 1} & & 0.296 & 0.300 & 0.519 & 0.500 & 0.579 & 0.600 & 0.736 & 0.658 \\
\hline & $\begin{array}{l}\left(x_{i}\right) \\
(0 / 0)\end{array}$ & 0.311 & 0.288 & 0.486 & 0.495 & 0.604 & 0.604 & 0.633 & 0.655 \\
\hline & & 0.297 & 0.298 & 0.518 & 0.484 & 0.593 & 0.590 & 0.604 & 0.670 \\
\hline & $\begin{array}{c}(\bar{x} \pm \delta) \\
(\%)\end{array}$ & $\begin{array}{c}0.301 \pm \\
0.008\end{array}$ & $\begin{array}{c}0.295 \pm \\
0.006\end{array}$ & $\begin{array}{c}0.507 \pm \\
0.02\end{array}$ & $\begin{array}{c}0.493 \pm \\
0.008\end{array}$ & $\begin{array}{c}0.593 \pm \\
0.01\end{array}$ & $\begin{array}{c}0.598 \pm \\
0.007\end{array}$ & $\begin{array}{c}0.658 \pm \\
0.07\end{array}$ & $\begin{array}{c}0.661 \pm \\
0.008\end{array}$ \\
\hline \multirow{4}{*}{ Mark 2} & & 0.270 & 0.263 & 0.466 & 0.477 & 0.592 & 0.562 & 0.616 & 0.607 \\
\hline & $\begin{array}{l}\left(x_{i}\right) \\
(0)\end{array}$ & 0.263 & 0.260 & 0.461 & 0.485 & 0.574 & 0.564 & 0.582 & 0.591 \\
\hline & & 0.262 & 0.251 & 0.528 & 0.490 & 0.551 & 0.578 & 0.589 & 0.596 \\
\hline & $\begin{array}{c}(\bar{x} \pm \delta) \\
(\%)\end{array}$ & $\begin{array}{c}0.265 \pm \\
0.004\end{array}$ & $\begin{array}{c}0.258 \pm \\
0.006\end{array}$ & $\begin{array}{c}0.485 \pm \\
0.04\end{array}$ & $\begin{array}{c}0.483 \pm \\
0.008\end{array}$ & $\begin{array}{c}0.572 \pm \\
0.02\end{array}$ & $\begin{array}{c}0.568 \pm \\
0.008\end{array}$ & $\begin{array}{c}0.595 \pm \\
0.02\end{array}$ & $\begin{array}{c}0.598 \pm \\
0.008\end{array}$ \\
\hline \multirow{4}{*}{ Mark 3} & & 0.250 & 0.253 & 0.440 & 0.425 & 0.508 & 0.493 & 0.547 & 0.516 \\
\hline & $\begin{array}{l}\left(x_{\mathrm{i}}\right) \\
(0)\end{array}$ & 0.261 & 0.255 & 0.439 & 0.435 & 0.496 & 0.504 & 0.529 & 0.548 \\
\hline & & 0.263 & 0.239 & 0.444 & 0.436 & 0.488 & 0.512 & 0.502 & 0.544 \\
\hline & $\begin{array}{c}(\bar{x} \pm \delta) \\
(\%)\end{array}$ & $\begin{array}{c}0.258 \pm \\
0.007\end{array}$ & $\begin{array}{c}0.249 \pm \\
0.08\end{array}$ & $\begin{array}{c}0.441 \pm \\
0.02\end{array}$ & $\begin{array}{c}0.432 \pm \\
0.006\end{array}$ & $\begin{array}{c}0.497 \pm \\
0.01\end{array}$ & $\begin{array}{c}0.503 \pm \\
0.009\end{array}$ & $\begin{array}{c}0.526 \pm \\
0.04\end{array}$ & $\begin{array}{c}0.536 \pm \\
0.02\end{array}$ \\
\hline \multirow{4}{*}{ Mark 4} & & 0.188 & 0.180 & 0.309 & 0.309 & 0.410 & 0.398 & 0.458 & \\
\hline & $\begin{array}{l}\left(\lambda_{i}\right) \\
(0 / 0)\end{array}$ & 0.178 & 0.190 & 0.329 & 0.290 & 0.401 & 0.396 & 0.432 & 0.415 \\
\hline & & 0.186 & 0.164 & 0.312 & 0.307 & 0.395 & 0.388 & 0.385 & 0.4180 .429 \\
\hline & $\begin{array}{c}(\bar{x} \pm \delta) \\
(\%)\end{array}$ & $\begin{array}{c}0.184 \pm \\
0.005\end{array}$ & $\begin{array}{c}0.178 \pm \\
0.01\end{array}$ & $\begin{array}{c}0.316 \pm \\
0.01\end{array}$ & $\begin{array}{c}0.302 \pm \\
0.01\end{array}$ & $\begin{array}{c}0.402 \pm \\
0.08\end{array}$ & $\begin{array}{c}0.394 \pm \\
0.005\end{array}$ & $\begin{array}{c}0.425 \pm \\
0.04\end{array}$ & $\begin{array}{c}0.420 \pm \\
0.008\end{array}$ \\
\hline
\end{tabular}

Dependence of arithmetic mean values of water soluble matter of exposure time [specimen H.S. (a) and specimen
I.R.S. (b)] for all four PR systems in synthetic sea water at $23^{\circ} \mathrm{C}$ for a period of thirty days are presented in Fig. 12 . 


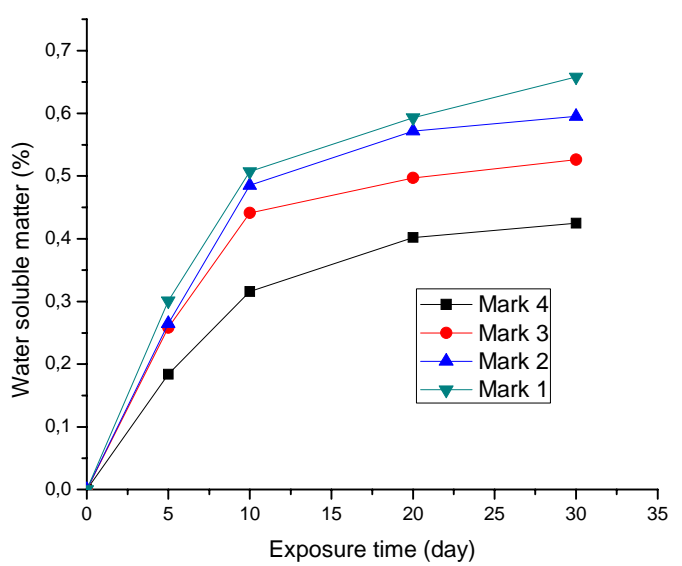

(a)

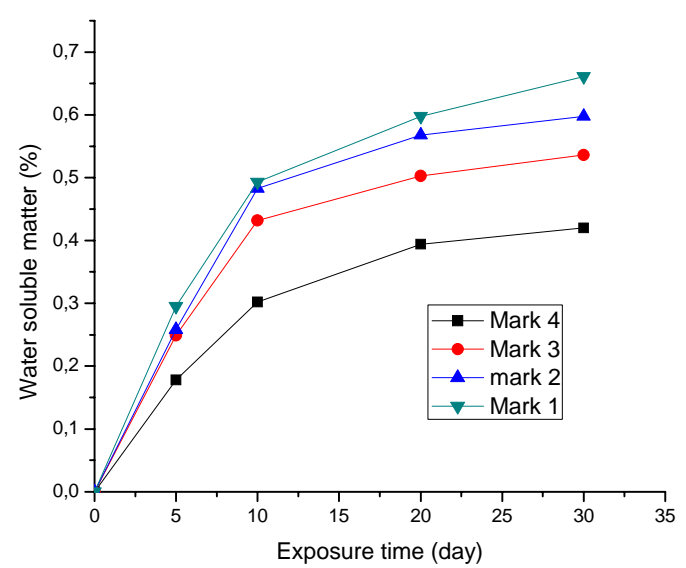

(b)

Figure 12. Dependence of water soluble matter of exposure time [specimen H.S. (a) and specimen I.R.S. (b)] for all four PR systems in synthetic sea water at $23{ }^{\circ} \mathrm{C}$ for a period of thirty days

Data recorded during drying one H.S. of each of four PR systems to constant mass, after immersing in distillated water (abbreviated for a table presentation in this paper-D.W.) and synthetic sea water (abbreviated for a table presentation in this paper-S.S.W.) at $23{ }^{\circ} \mathrm{C}$ for a period of thirty days, are presented in Table 7.

Table 7. Data recorded during drying one H.S. of each of four PR systems to constant mass, after immersing in distillated water (D.W.) and synthetic sea water (S.S.W.) at $23^{\circ} \mathrm{C}$ for a period of thirty days

\begin{tabular}{|c|c|c|c|c|c|c|c|c||}
\hline \hline \multirow{2}{*}{$\begin{array}{c}\text { Drying } \\
\text { time } \\
\text { (hour) }\end{array}$} & \multicolumn{9}{|c|}{ PR system mark } \\
\cline { 2 - 10 } & $\begin{array}{c}\text { After } \\
\text { D.W. } \\
\text { (g) }\end{array}$ & $\begin{array}{c}\text { After } \\
\text { S.S.W.(g) }\end{array}$ & $\begin{array}{c}\text { After } \\
\text { D.W. } \\
\text { (g) }\end{array}$ & $\begin{array}{c}\text { After } \\
\text { S.S.W.g) }\end{array}$ & $\begin{array}{c}\text { After } \\
\text { D.W. } \\
\text { (g) }\end{array}$ & $\begin{array}{c}\text { After } \\
\text { S.S.W.g) }\end{array}$ & $\begin{array}{c}\text { After } \\
\text { D.W. } \\
\text { (g) }\end{array}$ & $\begin{array}{c}\text { After } \\
\text { S.S.W.(g) }\end{array}$ \\
\hline \hline 0 & 7.7352 & 8.0104 & 7.2658 & 7.4276 & 8.3106 & 7.5114 & 7.7793 & 5.5785 \\
\hline 24 & 7.7142 & 7.9896 & 7.2431 & 7.4053 & 8.2919 & 7.4962 & 7.7662 & 5.5683 \\
\hline 48 & 7.6991 & 7.9772 & 7.2331 & 7.3953 & 8.2804 & 7.4871 & 7.7581 & 5.5615 \\
\hline 72 & 7.6908 & 7.9704 & 7.2280 & 7.3898 & 8.2747 & 7.4796 & 7.7525 & 5.5569 \\
\hline 96 & 7.6858 & 7.9669 & 7.2248 & 7.3868 & 8.2707 & 7.4752 & 7.7494 & 5.5541 \\
\hline 120 & 7.6825 & 7.9646 & 7.2231 & 7.3853 & 8.2689 & 7.4731 & 7.7484 & 5.5531 \\
\hline 144 & 7.6812 & 7.9633 & 7.2221 & 7.3847 & 8.2681 & 7.4720 & 7.7483 & 5.5530 \\
\hline 168 & 7.6802 & 7.9623 & 7.2220 & 7.3846 & 8.2680 & 7.4718 & $/$ & $/$ \\
\hline 192 & 7.6801 & 7.9622 & $/$ & $/$ & $/$ & $/$ & $/$ & $/$ \\
\hline \hline
\end{tabular}

As can be seen in Table 7, the longest drying time showed PR system mark 1 specimen, which is in accordance with the results of water absorption and water soluble matter of mentioned system. The shortest drying time showed PR system mark 4 specimens, also, based on values of two mentioned tested characteristics.

PR system mark 4 has the greatest resistance toward distillated and synthetic sea water due to basic components, including alcohol (neopenthyl glycol) and saturated acid (isophthalic acid) and synthesis procedure (the highest molecular weight of all tested polyester resin systems). The positive influence of neopentyl glycol to hydrolytic stability of PR system mark 4 is based on a fact that two $\mathrm{H}$ atoms of $\alpha$ carbon atom are replaced by two methyl groups. The greatest water resistances of PR system mark 4 means that this PR system showed the smallest both water absorption and water soluble matter.

From the practical point of view the worst water absorption value and water soluble matter value of four tested PR systems is recorded at PR system mark 1 . This phenomenon is caused by influence of diethylene glycol (because ether linkages in this constituent have increased water and heat sensitivity) and of ortho phthalic acid (which produces resin of lower molecular weight regarding isophthalic acid).

By the analysis of data presented in Tables 2 to 7 and Figures 9 to 12, which refers to water absorption and water soluble matter in distillated water and synthetic sea water, it can be seen that the results obtained by testing hardness specimens and impact resistance specimens are similar. This fact indicates that the procedure of manufacturing of mentioned specimens and procedure of testing hardness specimens and impact resistance specimens, after immersing in distillated water and synthetic sea water, were done correctly.

Barcol hardness [single values $\left(\mathrm{x}_{\mathrm{i}}\right)$ and adopted value for later analysis], expressed in Barcol degree (abbreviated for a table presentation in this paper-B.D.) of H.S. of each of four PR systems, after immersing in distillated water and synthetic sea water at $23{ }^{\circ} \mathrm{C}$ for a periods $5,10,20$ and 30 days, are presented in Table 8.

Table 8. Barcol hardness of H.S. of each of four PR systems, after immersing in distillated water and synthetic sea water at $23{ }^{\circ} \mathrm{C}$ for the periods of $5,10,20$ and 30 days

\begin{tabular}{|c|c|c|c|c|c|c|c|}
\hline \multirow{2}{*}{$\begin{array}{c}\text { PR system } \\
\text { mark }\end{array}$} & \multirow{2}{*}{\multicolumn{2}{|c|}{$\begin{array}{c}\text { Liquid to which H.S. } \\
\text { was exposed }\end{array}$}} & \multicolumn{5}{|c|}{ Exposure time (day) } \\
\hline & & & 0 & 5 & 10 & 20 & 30 \\
\hline \multirow{4}{*}{ Mark 1} & \multirow{2}{*}{ D.W. } & \begin{tabular}{|c|} 
Single values \\
(B.D.)
\end{tabular} & $42,41,42$ & $40,41,40$ & $37,36,36$ & $35,34,34$ & $32,33,32$ \\
\hline & & $\begin{array}{c}\text { Adopted value } \\
\text { (B.D.) } \\
\end{array}$ & 42 & 40 & 36 & 34 & 32 \\
\hline & \multirow{2}{*}{ S.S.W. } & \begin{tabular}{|c|} 
Single values \\
(B.D.)
\end{tabular} & $42,41,42$ & $41,40,41$ & $38,39,38$ & $36,36,37$ & $34,35,34$ \\
\hline & & $\begin{array}{c}\text { Adopted value } \\
\text { (B.D.) }\end{array}$ & 42 & 41 & 38 & 36 & 34 \\
\hline
\end{tabular}




\begin{tabular}{|c|c|c|c|c|c|c|c|}
\hline \multirow{4}{*}{ Mark 2} & \multirow{2}{*}{ D.W. } & $\begin{array}{c}\text { Single values } \\
\text { (B.D.) }\end{array}$ & $42,42,42$ & $41,42,41$ & $41,41,40$ & $40,40,40$ & $39,40,39$ \\
\hline & & $\begin{array}{l}\text { Adopted value } \\
\text { (B.D.) }\end{array}$ & 42 & 41 & 41 & 40 & 39 \\
\hline & \multirow{2}{*}{ S.S.W. } & $\begin{array}{l}\text { Single values } \\
\text { (B.D.) }\end{array}$ & $42,42,42$ & $41,42,41$ & $41,41,41$ & $41,40,41$ & $40,40,40$ \\
\hline & & $\begin{array}{l}\text { Adopted value } \\
\text { (B.D.) }\end{array}$ & 42 & 41 & 41 & 41 & 40 \\
\hline \multirow{4}{*}{ Mark 3} & \multirow{2}{*}{ D.W. } & $\begin{array}{l}\text { Single values } \\
\text { (B.D.) }\end{array}$ & $44,43,44$ & $44,43,43$ & $43,42,43$ & $42,42,42$ & $41,42,41$ \\
\hline & & $\begin{array}{l}\text { Adopted value } \\
\text { (B.D.) }\end{array}$ & 44 & 43 & 43 & 42 & 41 \\
\hline & \multirow{2}{*}{ S.S.W. } & $\begin{array}{l}\text { Single values } \\
\text { (B.D.) }\end{array}$ & $44,43,44$ & $44,43,43$ & $43,42,43$ & $42,43,42$ & $41,42,41$ \\
\hline & & $\begin{array}{l}\text { Adopted value } \\
\text { (B.D.) }\end{array}$ & 44 & 43 & 43 & 42 & 41 \\
\hline \multirow{4}{*}{ Mark4 } & \multirow{2}{*}{ D.W. } & $\begin{array}{l}\text { Single values } \\
\text { (B.D.) }\end{array}$ & $41,42,41$ & $40,41,40$ & $40,39,39$ & $38,37,38$ & $37,36,37$ \\
\hline & & $\begin{array}{c}\text { Adopted value } \\
\text { (B.D.) }\end{array}$ & 41 & 40 & 39 & 38 & 37 \\
\hline & \multirow{2}{*}{ S.S.W. } & $\begin{array}{l}\text { Single values } \\
\text { (B.D.) }\end{array}$ & $41,42,41$ & $41,40,40$ & $40,39,40$ & $39,38,39$ & $38,38,37$ \\
\hline & & $\begin{array}{l}\text { Adopted value } \\
\text { (B.D.) }\end{array}$ & 41 & 40 & 40 & 39 & 38 \\
\hline
\end{tabular}

Barcol hardness (adopted values) of H.S. of four PR systems, after immersing in distillated water (a) and synthetic

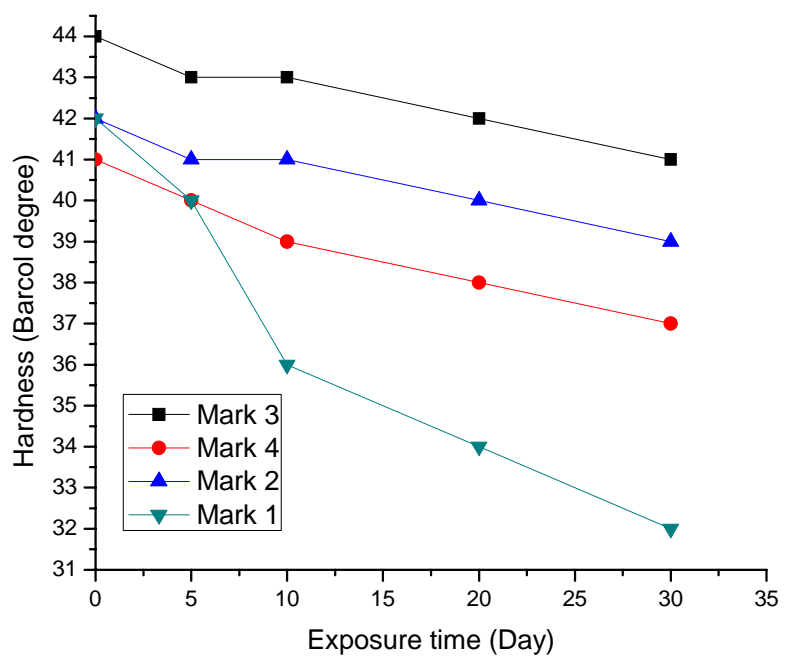

(a) sea water (b) at $23{ }^{\circ} \mathrm{C}$ for the periods of 5, 10, 20 and 30 days, are presented in Fig.13.

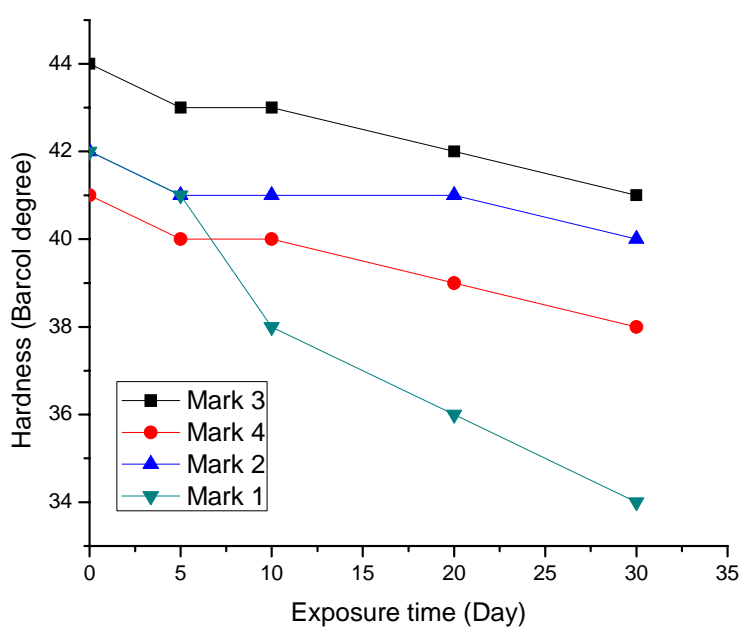

(b)

Figure 13. Barcol hardness (adopted value) of H.S. of four PR systems, after immersing in distillated water (a) and synthetic sea water (b) at $23^{\circ} \mathrm{C}$ for the periods of $5,10,20$ and 30 days

All four PR systems have similar initial Barcol hardness. By analyzing Barcol hardness data, obtained after the influence of distillated and synthetic sea water at the laboratory temperature $\left(23^{\circ} \mathrm{C}\right)$ for a period of 30 days, it can be seen, nominally, that highest decrease is shown by PS system mark 1 (from $20 \%$ to $25 \%$ ). PR systems marks 2 and 3 have similar Barcol hardness drop (about $7 \%$ ) and PS system mark 4 something higher (about $10 \%$ ).

Based on Barcol hardness results for PR system mark 2, PR system mark 3 and PR system mark 4, after immersion in distillated and synthetic sea water for 5,10, 20 and 30 days and for PR system mark 1, after immersion in distillated and synthetic sea water for 5 days, one may conclude that a

Table 9. Impact resistance of I.R.S. of four PR systems, after immersing in distillated water and synthetic sea water at $23{ }^{\circ} \mathrm{C}$ for the periods of $5,10,20$ and 30 days

\begin{tabular}{|c|c|c|c|c|c|c|c|}
\hline \multirow{2}{*}{$\begin{array}{l}\text { PR system } \\
\text { mark }\end{array}$} & \multirow{2}{*}{\multicolumn{2}{|c|}{$\begin{array}{l}\text { Liquid to which } \\
\text { I.R.S. } \\
\text { was exposed } \\
\end{array}$}} & \multicolumn{5}{|c|}{ Exposure time (day) } \\
\hline & & & 0 & 5 & 10 & 20 & 30 \\
\hline \multirow[t]{2}{*}{ Mark 1} & \multirow{2}{*}{ D.W. } & $\begin{array}{c}\left(\mathrm{x}_{\mathrm{i}}\right) \\
\left(\mathrm{kJ} / \mathrm{m}^{2}\right)\end{array}$ & $\begin{array}{c}5.00 ; 5.21 \\
5.12\end{array}$ & $\begin{array}{c}5.08 ; 5.22 \\
5.30\end{array}$ & $\begin{array}{c}5.48 ; \\
5.31 ; 5.44\end{array}$ & $\begin{array}{c}5.20 ; \\
5.35 ; 5.32\end{array}$ & $\begin{array}{c}5.09 ; 4.92 \\
5.04\end{array}$ \\
\hline & & $\begin{array}{l}(\bar{x} \pm \delta) \\
\left(\mathrm{kJ} / \mathrm{m}^{2}\right)\end{array}$ & $5.11 \pm 0.10$ & $5.20 \pm 0.11$ & $5.41 \pm 0.09$ & $5.29 \pm 0.08$ & $5.02 \pm 0.09$ \\
\hline
\end{tabular}

plasticization process started slightly. Important drop of Barcol hardness for PR system mark 1, after immersion in distillated and synthetic sea water for 10, 20 and 30 days, can be an indication of beginning of swelling and breaking bonds processes in surface layer.

Impact resistance [single values $\left(\mathrm{x}_{\mathrm{i}}\right)$ and arithmetic mean value $(\bar{x} \pm \delta)$ ] of I.R.S. of each of four PR systems, after immersing in distillated water and synthetic sea water at $23{ }^{\circ} \mathrm{C}$ for periods of 5, 10, 20 and 30 days, is presented in Table 9. 


\begin{tabular}{|c|c|c|c|c|c|c|c|}
\hline & SI & $\begin{array}{c}\left(\mathrm{x}_{\mathrm{i}}\right) \\
\left(\mathrm{kJ} / \mathrm{m}^{2}\right)\end{array}$ & $\begin{array}{l}5.00 ; 5.21 ; \\
5.12\end{array}$ & $\begin{array}{l}5.02 ; 5.08 \\
5.20\end{array}$ & $\begin{array}{l}5.22 ; 5.34 \\
5.40\end{array}$ & $\begin{array}{l}5.28 ; 5.11 \\
5.24\end{array}$ & $\begin{array}{c}4.89 ; 4.82 \\
4.93\end{array}$ \\
\hline & S.S.W & $\begin{array}{l}(\bar{x} \pm \delta) \\
\left(\mathrm{kJ} / \mathrm{m}^{2}\right)\end{array}$ & $5.11 \pm 0.10$ & $5.10 \pm 0.09$ & $5.32 \pm 0.09$ & $5.21 \pm 0.08$ & $4.88 \pm 0.05$ \\
\hline \multirow{4}{*}{ Mark 2} & \multirow{2}{*}{ D.W. } & $\begin{array}{c}\left(\mathrm{x}_{\mathrm{i}}\right) \\
\left(\mathrm{kJ} / \mathrm{m}^{2}\right)\end{array}$ & $\begin{array}{c}16.83 ; 17.01 \\
16.54\end{array}$ & $\begin{array}{c}17.41 ; 17.06 \\
17.16\end{array}$ & $\begin{array}{c}17.58 ; 17.50 \\
17.36\end{array}$ & $\begin{array}{c}17.01 ; 17.53 ; \\
17.11\end{array}$ & $\begin{array}{c}16.98 ; 16.90 \\
16.76\end{array}$ \\
\hline & & $\begin{array}{l}(\bar{x} \pm \delta) \\
\left(\mathrm{kJ} / \mathrm{m}^{2}\right)\end{array}$ & $16.79 \pm 0.24$ & $17.21 \pm 0.18$ & $17.48 \pm 0.12$ & $17.22 \pm 0.28$ & $16.88 \pm 0.11$ \\
\hline & \multirow{2}{*}{ S.S.W. } & $\begin{array}{c}\left(\mathrm{x}_{\mathrm{i}}\right) \\
\left(\mathrm{kJ} / \mathrm{m}^{2}\right)\end{array}$ & $\begin{array}{c}16.83 ; 17.01 ; \\
16.54 \\
\end{array}$ & $\begin{array}{c}17.31 ; 17.06 \\
16.96 \\
\end{array}$ & $\begin{array}{c}17.45 ; 17.37 \\
17.21 \\
\end{array}$ & $\begin{array}{c}16.92 ; 17.01 ; \\
16.77\end{array}$ & $\begin{array}{c}16.53 ; 16.58 \\
16.33 \\
\end{array}$ \\
\hline & & $\begin{array}{c}(\bar{x} \pm \delta) \\
\left(\mathrm{kJ} / \mathrm{m}^{2}\right)\end{array}$ & $16.79 \pm 0.24$ & $17.11 \pm 0.18$ & $17.34 \pm 0.12$ & $16.92 \pm 0.13$ & $16.48 \pm 0.13$ \\
\hline \multirow{4}{*}{ Mark 3} & \multirow{2}{*}{ D.W. } & $\begin{array}{c}\left(\mathrm{x}_{\mathrm{i}}\right) \\
\left(\mathrm{kJ} / \mathrm{m}^{2}\right)\end{array}$ & $\begin{array}{c}7.44 ; 6.81 ; \\
\quad 6.72\end{array}$ & $\begin{array}{c}7.53 ; 7.58 ; \\
7.33\end{array}$ & $\begin{array}{c}7.33 ; 7.37 \\
7.19 \\
\end{array}$ & $\begin{array}{c}7.16 ; 7.11 ; \\
7.36\end{array}$ & $\begin{array}{c}7.24 ; 7.02 \\
7.10\end{array}$ \\
\hline & & $\begin{array}{l}(\bar{x} \pm \delta) \\
\left(\mathrm{kJ} / \mathrm{m}^{2}\right)\end{array}$ & $6.99 \pm 0.39$ & $7.48 \pm 0.12$ & $7.29 \pm 0.09$ & $7.21 \pm 0.13$ & $7.12 \pm 0.11$ \\
\hline & \multirow{2}{*}{ S.S.W. } & $\begin{array}{c}\left(\mathrm{x}_{\mathrm{i}}\right) \\
\left(\mathrm{kJ} / \mathrm{m}^{2}\right)\end{array}$ & $\begin{array}{c}7.44 ; 6.81 \\
\quad 6.72\end{array}$ & $\begin{array}{c}7.22 ; 7.27 \\
7.47\end{array}$ & $\begin{array}{c}7.35 ; 7.00 \\
7.05\end{array}$ & $\begin{array}{l}6.99 ; 7.12 ; \\
\quad 6.65\end{array}$ & $\begin{array}{c}6.51 ; 6.63 \\
6.34\end{array}$ \\
\hline & & $\begin{array}{l}(\bar{x} \pm \delta) \\
\left(\mathrm{kJ} / \mathrm{m}^{2}\right)\end{array}$ & $6.99 \pm 0.39$ & $7.32 \pm 0.13$ & $7.13 \pm 0.19$ & $6.92 \pm 0.24$ & $6.49 \pm 0.15$ \\
\hline \multirow{4}{*}{ Mark 4} & \multirow{2}{*}{ D.W. } & $\begin{array}{c}\left(\mathrm{x}_{\mathrm{i}}\right) \\
\left(\mathrm{kJ} / \mathrm{m}^{2}\right)\end{array}$ & $\begin{array}{c}14.62 ; 14.91 \\
15.22\end{array}$ & $\begin{array}{c}15.22 ; 15.27 \\
15.47\end{array}$ & $\begin{array}{c}15.88 ; 15.50 \\
15.65\end{array}$ & $\begin{array}{c}14.99 ; 15.02 \\
14.75\end{array}$ & $\begin{array}{c}14.78 ; 14.40 ; \\
14.55\end{array}$ \\
\hline & & $\begin{array}{l}(\bar{x} \pm \delta) \\
\left(\mathrm{kJ} / \mathrm{m}^{2}\right)\end{array}$ & $14.92 \pm 0.30$ & $15.32 \pm 0.13$ & $15.68 \pm 0.19$ & $14.92 \pm 0.15$ & $14.58 \pm 0.19$ \\
\hline & \multirow{2}{*}{ S.S.W. } & $\begin{array}{c}\left(\mathrm{x}_{\mathrm{i}}\right) \\
\left(\mathrm{kJ} / \mathrm{m}^{2}\right)\end{array}$ & $\begin{array}{c}14.62 ; 14.91 ; \\
15.22\end{array}$ & $\begin{array}{c}15.58 ; 14.80 \\
15.15\end{array}$ & $\begin{array}{c}15.68 ; 15.40 \\
15.56\end{array}$ & $\begin{array}{c}14.70 ; 14.76 \\
14.65\end{array}$ & $\begin{array}{c}13.94 ; 13.99 \\
14.00\end{array}$ \\
\hline & & $\begin{array}{c}(\bar{x} \pm \delta) \\
\left(\mathrm{kJ} / \mathrm{m}^{2}\right)\end{array}$ & $14.92 \pm 0.30$ & $15.10 \pm 0.34$ & $15.55 \pm 0.14$ & $14.70 \pm 0.05$ & $13.98 \pm 0.03$ \\
\hline
\end{tabular}

Impact resistance of I.R.S. of four PR systems, after immersing in distillated water (a) and synthetic sea water (b)

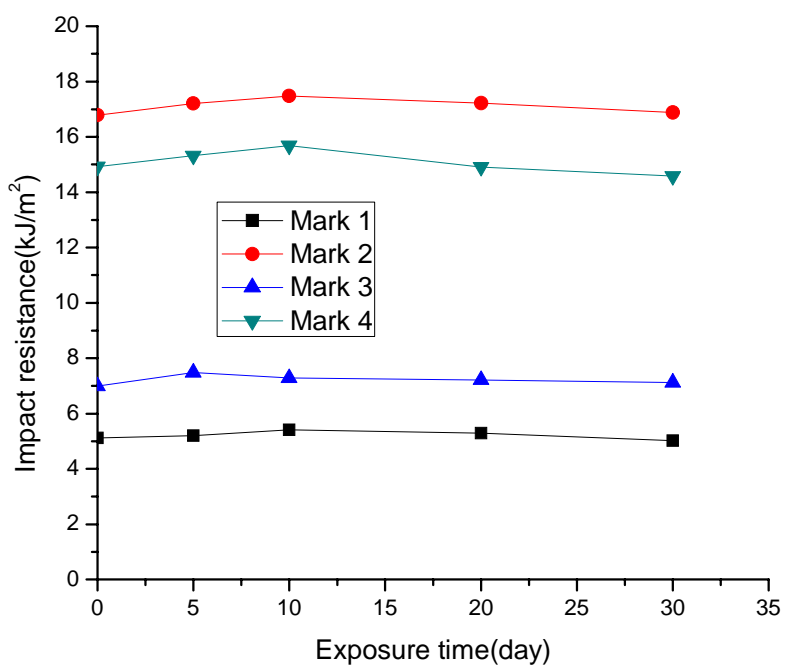

(a) at $23{ }^{\circ} \mathrm{C}$ for the periods of $5,10,20$ and 30 days, is presented in Fig. 14.

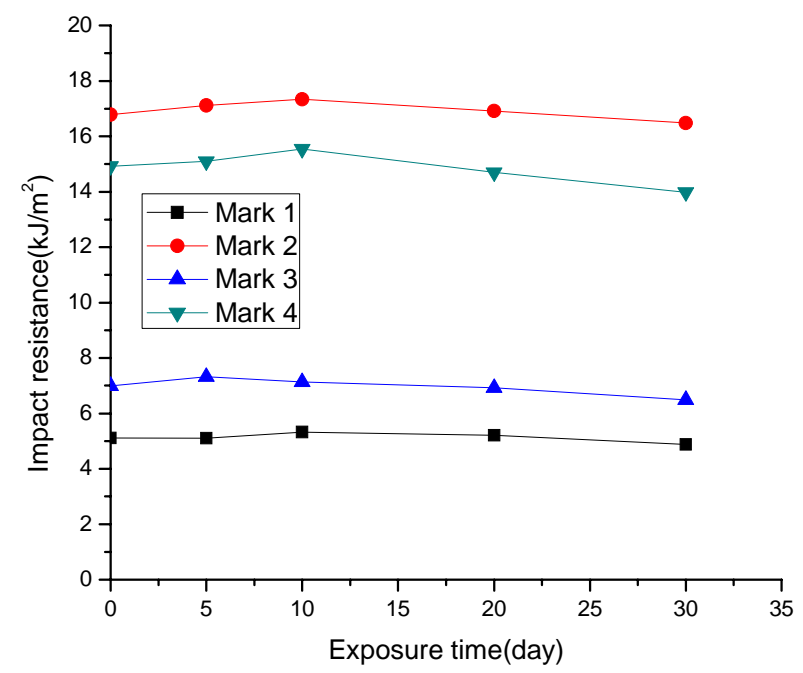

(b)

Figure 14. Impact resistance of I.R.S. of four PR systems, after immersing in distillated water (a) and synthetic sea water (b) at $23{ }^{\circ} \mathrm{C}$ for the periods of $5,10,20$ and 30 days

Impact resistance results can be broadly divided into two groups:

- in the first are PR systems marks 2 and 4, whose initial impact resistance is 16.79 and $14.92 \mathrm{~kJ} / \mathrm{m}^{2}$, respectively and

- in the second are PR systems marks 3 and 1, whose initial impact resistance is 6.99 and $5.11 \mathrm{~kJ} / \mathrm{m}^{2}$, respectively.

PR systems from the first group have branched alcohol in their composition.

PR system mark 2 has pentaerytritol, which introduces branching in the polyester chain and improves mentioned physical property and resistance toward distillated and synthetic sea water. High molecular weight of this system, also, has a positive influence to the tested characteristics.

Neopentyl glycol, present in PR system mark 4, primarily provides high impact resistance and, obviously, improves water resistance although isophthalic acid and high molecular weight, also, have positive influence on the mentioned characteristics.

\section{Testing results with boiling water and analysis}

One specimen dimensions $25 \mathrm{~mm} \times 15 \mathrm{~mm} \times 10 \mathrm{~mm}$ and one specimen dimensions $120 \mathrm{~mm} \times 15 \mathrm{~mm} \times 10 \mathrm{~mm}$ of each of four PR systems were immersed in boiling distillated water for a 
period of 210 minutes. Data recorded during this testing and

water soluble matter (single value), are presented in Table 10. calculated corresponding water absorption (single value) and

Table 10. Data recorded during testing one H.S. and one I.R.S. of each of four PR systems in boiling distillated water for a period up to 210 minutes and corresponding water absorption value and water soluble matter value

\begin{tabular}{|c|c|c|c|c|c|c|c|c|}
\hline \multirow{3}{*}{ Parameters } & \multicolumn{8}{|c|}{ PR system mark } \\
\hline & \multicolumn{2}{|c|}{ Mark 1} & \multicolumn{2}{|c|}{ Mark 2} & \multicolumn{2}{|c|}{ Mark 3} & \multicolumn{2}{|c|}{ Mark 4} \\
\hline & H.S. & I.R.S. & H.S. & I.R.S. & H.S. & I.R.S. & H.S. & I.R.S. \\
\hline$m_{1}(\mathrm{~g})$ & 6.5101 & 18.3225 & 6.4501 & 19.8563 & 7.9336 & 19.5236 & 6.5844 & 18.1425 \\
\hline$m_{2,30, \text { b.w. }}(\mathrm{g})$ & 6.5282 & 18.3723 & 6.4643 & 19.8960 & 7.9513 & 19.5646 & 6.5949 & 18.1706 \\
\hline$C_{\text {a.w. }}(\%)$ & 0.278 & 0.272 & 0.220 & 0.205 & 0.223 & 0.210 & 0.159 & 0.155 \\
\hline$m_{3,30, \text { b.w.,c.m.(g) }}$ & 6.4980 & 18.2862 & 6.4431 & 19.8336 & 7.9269 & 19.5080 & 6.5792 & 18.1279 \\
\hline$C_{\text {w.s.m. }}(\%)$ & 0.463 & 0.470 & 0.328 & 0.314 & 0.307 & 0.290 & 0.238 & 0.235 \\
\hline$m_{1}(\mathrm{~g})$ & 6.5101 & 18.3225 & 6.4501 & 19.8563 & 7.9336 & 19.5236 & 6.5844 & 18.1425 \\
\hline$m_{2,60 \text {,b.w. }}(\mathrm{g})$ & 6.5360 & 19.3912 & 6.4697 & 19.9099 & 7.9565 & 19.5773 & 6.6003 & 18.1846 \\
\hline $\mathrm{C}_{\text {a.w.w }}(\%)$ & 0.398 & 0.375 & 0.304 & 0.270 & 0.288 & 0.275 & 0.241 & 0.232 \\
\hline$m_{3,60, \text { b.w.c.c.m.(g) }}$ & 6.4974 & 18.2827 & 6.4424 & 19.8265 & 7.9250 & 19.5002 & 6.5780 & 18.1263 \\
\hline$C_{\text {w.s.m. }}(\%)$ & 0.593 & 0.592 & 0.422 & 0.420 & 0.396 & 0.395 & 0.339 & 0.321 \\
\hline $\mathrm{m}_{1}(\mathrm{~g})$ & 6.5101 & 18.3225 & 6.4501 & 19.8563 & 7.9336 & 19.5236 & 6.5844 & 18.1425 \\
\hline$m_{2,90, \text { b.w. }}(\mathrm{g})$ & 6.5414 & 18.4068 & 6.4743 & 19.9329 & 7.9607 & 19.5942 & 6.6038 & 18.1942 \\
\hline $\mathrm{C}_{\mathrm{a} . \mathrm{w}} \cdot(\%)$ & 0.481 & 0.460 & 0.375 & 0.383 & 0.341 & 0.362 & 0.295 & 0.285 \\
\hline$m_{3,90, \text { b.w.c.c.m.. }(\mathrm{g})}$ & 6.4970 & 18.2849 & 6.4423 & 19.8380 & 7.9230 & 19.4522 & 6.5775 & 18.1223 \\
\hline$C_{\text {w.s.m. }}(\%)$ & 0.682 & 0.665 & 0.495 & 0.478 & 0.475 & 0.460 & 0.399 & 0.396 \\
\hline$m_{1}(\mathrm{~g})$ & 6.5101 & 18.3225 & 6.4501 & 19.8563 & 7.9336 & 19.5236 & 6.5844 & 18.1425 \\
\hline$m_{2,120, \text { b.w. }}(\mathrm{g})$ & 6.5473 & 18.4223 & 6.4780 & 19.9416 & 7.9643 & 19.6034 & 6.6062 & 18.2014 \\
\hline $\mathrm{C}_{\mathrm{a} . \mathrm{w} \cdot(\%)}$ & 0.574 & 0.545 & 0.432 & 0.430 & 0.387 & 0.409 & 0.331 & 0.325 \\
\hline$m_{3,120, \text { b.w.,c.m. } .(\mathrm{g})}$ & 6.4977 & 18.2830 & 6.4415 & 19.8304 & 7.9328 & 19.5017 & 6.5755 & 18.1188 \\
\hline$C_{\text {w.s.m. }}(\%)$ & 0.762 & 0.760 & 0.566 & 0.560 & 0.530 & 0.521 & 0.465 & 0.455 \\
\hline$m_{1}(\mathrm{~g})$ & 6.5101 & 18.3225 & 6.4501 & 19.8563 & 7.9336 & 19.5236 & 6.5844 & 18.1425 \\
\hline$m_{2,150, \text { b.w. }}(\mathrm{g})$ & 6.5511 & 18.4342 & 6.4817 & 19.9506 & 7.9684 & 19.6153 & 6.6085 & 18.2085 \\
\hline$C_{\text {a.w. }}(\%)$ & 0.630 & 0.610 & 0.490 & 0.475 & 0.439 & 0.470 & 0.366 & 0.364 \\
\hline$\left.m_{3,150, \text { b.w.,c.m. }} . \mathrm{g}\right)$ & 6.4970 & 18.2845 & 6.4410 & 19.8255 & 7.9208 & 19.4985 & 6.5728 & 18.1105 \\
\hline$C_{\text {w.s.m. }}(\%)$ & 0.830 & 0.817 & 0.630 & 0.630 & 0.600 & 0.598 & 0.542 & 0.540 \\
\hline$m_{1}(\mathrm{~g})$ & 6.5101 & 18.3225 & 6.4501 & 19.8563 & 7.9336 & 19.5236 & 6.5844 & 18.1425 \\
\hline$m_{2,180, \text { b.w. }}(\mathrm{g})$ & 6.5546 & 18.4396 & 6.4845 & 19.9615 & 7.9718 & 19.6192 & 6.6111 & 18.2143 \\
\hline$C_{\text {a.w. }}(\%)$ & 0.683 & 0.639 & 0.533 & 0.530 & 0.481 & 0.490 & 0.405 & 0.396 \\
\hline$m_{3,180, \text { b.w.,c.m. }(\mathrm{g})}$ & 6.4970 & 18.2802 & 6.4395 & 19.8235 & 7.9198 & 19.4954 & 6.5717 & 18.1054 \\
\hline$C_{\text {w.s.m. }}(\%)$ & 0.885 & 0.870 & 0.697 & 0.695 & 0.655 & 0.634 & 0.598 & 0.600 \\
\hline$m_{1}(\mathrm{~g})$ & 6.5101 & 18.3225 & 6.4501 & 19.8563 & 7.9336 & 19.5236 & 6.5844 & 18.1425 \\
\hline$m_{2,210, \text { b.w. }}(\mathrm{g})$ & 6.5561 & 18.4471 & 6.4872 & 19.9695 & 7.9742 & 19.6308 & 6.6131 & 18.2214 \\
\hline$C_{\text {a.w. }}(\%)$ & 0.706 & 0.680 & 0.575 & 0.570 & 0.512 & 0.549 & 0.436 & 0.435 \\
\hline$m_{3,210, \text { b.w.,c.m. } .(\mathrm{g})}$ & 6.4960 & 18.2805 & 6.4380 & 19.8219 & 7.9166 & 19.4923 & 6.5716 & 18.1085 \\
\hline$C_{\text {w.s.m. }}(\%)$ & 0.922 & 0.909 & 0.752 & 0.743 & 0.726 & 0.709 & 0.630 & 0.622 \\
\hline
\end{tabular}

Data recorded during testing water absorption of specimens marks H.S. and I.R.S. of all four mentioned PR systems in boiling water for the periods of $30,60,90,120,150,180$ and
210 minutes [single values $\left(x_{\mathrm{i}}\right)$ and arithmetic mean value $(\bar{x}$ $\pm \delta)$ ] are presented in Table 11 .

Table 11. Single values $\left(\mathrm{x}_{\mathrm{i}}\right)$ and arithmetic mean value $(\bar{x} \pm \delta)$ for water absorption of specimens marks H.S. and I.R.S. of all four mentioned PR systems in boiling water for the periods of $30,60,90,120,150,180$ and 210 minutes

\begin{tabular}{|c|c|c|c|c|c|c|c|c|c|}
\hline \multirow{3}{*}{\multicolumn{2}{|c|}{$\begin{array}{l}\text { Exposure time } \\
\text { (minute) }\end{array}$}} & \multicolumn{8}{|c|}{ PR system mark } \\
\hline & & \multicolumn{2}{|c|}{ Mark 1} & \multicolumn{2}{|c|}{ Mark 2} & \multicolumn{2}{|c|}{ Mark 3} & \multicolumn{2}{|c|}{ Mark 4} \\
\hline & & H.S. & I.R.S. & H.S. & I.R.S. & H.S. & I.R.S. & H.S. & I.R.S. \\
\hline \multirow{4}{*}{30} & & 0.278 & 0.272 & 0.220 & 0.205 & 0.223 & 0.210 & 0.159 & 0.155 \\
\hline & $\left(\mathrm{X}_{\mathrm{i}}\right)$ & 0.271 & 0.268 & 0.223 & 0.222 & 0.218 & 0.190 & 0.180 & 0.172 \\
\hline & & 0.268 & 0.255 & 0.226 & 0.203 & 0.195 & 0.203 & 0.178 & 0.168 \\
\hline & $\begin{array}{c}(\bar{x} \pm \delta) \\
(\%)\end{array}$ & $\begin{array}{c}0.272 \\
\pm 0.005\end{array}$ & $\begin{array}{l}0.265 \\
\pm 0.01\end{array}$ & $\begin{array}{c}0.223 \\
\pm 0.003\end{array}$ & $\begin{array}{l}0.210 \\
\pm 0.01\end{array}$ & $\begin{array}{l}0.212 \\
\pm 0.01\end{array}$ & $\begin{array}{l}0.201 \\
\pm 0.01\end{array}$ & $\begin{array}{l}0.172 \\
\pm 0.01\end{array}$ & $\begin{array}{c}0.165 \\
\pm 0.009\end{array}$ \\
\hline \multirow{4}{*}{60} & & 0.398 & 0.375 & 0.304 & 0.270 & 0.288 & 0.275 & 0.241 & 0.232 \\
\hline & $\begin{array}{l}\left(x_{i}\right) \\
(0 / 4)\end{array}$ & 0.390 & 0.380 & 0.313 & 0.290 & 0.297 & 0.284 & 0.248 & 0.230 \\
\hline & & 0.390 & 0.388 & 0.283 & 0.295 & 0.301 & 0.282 & 0.245 & 0.243 \\
\hline & $\begin{array}{c}(\bar{x} \pm \delta) \\
(\%)\end{array}$ & $\begin{array}{c}0.392 \\
\pm 0.004\end{array}$ & $\begin{array}{c}0.381 \\
\pm 0.006\end{array}$ & $\begin{array}{c}0.308 \\
\pm 0.006\end{array}$ & $\begin{array}{l}0.285 \\
\pm 0.01\end{array}$ & $\begin{array}{c}0.295 \\
\pm 0.006\end{array}$ & $\begin{array}{c}0.280 \pm \\
0.005\end{array}$ & $\begin{array}{c}0.244 \\
\pm 0.003\end{array}$ & $\begin{array}{l}0.235 \\
\pm 0.07\end{array}$ \\
\hline
\end{tabular}




\begin{tabular}{|c|c|c|c|c|c|c|c|c|c|}
\hline \multirow{4}{*}{90} & \multirow{3}{*}{$\begin{array}{l}\left(\mathrm{x}_{\mathrm{i}}\right) \\
(\%)\end{array}$} & 0.481 & 0.460 & 0.375 & 0.386 & 0.341 & 0.362 & 0.295 & 0.285 \\
\hline & & 0.461 & 0.480 & 0.387 & 0.370 & 0.373 & 0.358 & 0.302 & 0.293 \\
\hline & & 0.470 & 0.455 & 0.381 & 0.372 & 0.388 & 0.345 & 0.292 & 0.295 \\
\hline & $(\bar{x} \pm \delta)$ & $\begin{array}{l}0.471 \\
+0.01\end{array}$ & $\begin{array}{l}0.465 \\
\pm 0.01\end{array}$ & $\begin{array}{c}0.381 \\
\pm 0.006\end{array}$ & $\begin{array}{c}0.376 \\
+0.008\end{array}$ & $\begin{array}{l}0.367 \\
+0.02\end{array}$ & $\begin{array}{c}0.355 \\
+0.00\end{array}$ & $\begin{array}{c}0.297 \\
\pm 0.004\end{array}$ & $\begin{array}{c}0.291 \\
\pm 0.005\end{array}$ \\
\hline \multirow{5}{*}{120} & & 0.574 & 0.545 & 0.432 & 0.430 & 0.387 & 0.409 & 0.331 & 0.325 \\
\hline & $\left(x_{i}\right)$ & 0.552 & 0.559 & 0.443 & 0.411 & 0.418 & 0.405 & 0.338 & 0.342 \\
\hline & & 0.559 & 0.548 & 0.438 & 0.434 & 0.441 & 0.392 & 0.343 & 0.323 \\
\hline & $(\bar{x} \pm \delta)$ & 0.562 & 0.551 & 0.437 & 0.425 & 0.415 & 0.402 & 0.337 & 0.330 \\
\hline & 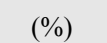 & \pm 0.01 & \pm 0.007 & \pm 0.005 & \pm 0.01 & \pm 0.03 & \pm 0.009 & \pm 0.006 & \pm 0.01 \\
\hline \multirow{5}{*}{150} & & 0.630 & $\begin{array}{l}0.610 \\
\end{array}$ & 0.490 & 0.475 & 0.439 & 0.470 & 0.366 & 0.364 \\
\hline & & 0.609 & 0.609 & 0.493 & 0.480 & 0.475 & 0.462 & 0.374 & 0.375 \\
\hline & & 0.615 & 0.590 & 0.496 & 0.488 & 0.490 & 0.463 & 0.377 & 0.355 \\
\hline & $(\bar{x} \pm \delta)$ & 0.618 & 0.603 & 0.493 & 0.481 & 0.470 & 0.465 & 0.372 & 0.365 \\
\hline & $(\%)$ & \pm 0.01 & \pm 0.01 & \pm 0.003 & \pm 0.006 & \pm 0.03 & \pm 0.004 & \pm 0.005 & \pm 0.01 \\
\hline \multirow{5}{*}{180} & & 0.683 & $\begin{array}{l}0.639 \\
\end{array}$ & 0.533 & 0.530 & 0.481 & $\begin{array}{l}0.490 \\
\end{array}$ & 0.405 & 0.396 \\
\hline & $\left(\mathrm{x}_{\mathrm{i}}\right.$ & 0.629 & 0.641 & 0.541 & 0.535 & 0.526 & 0.508 & 0.407 & 0.409 \\
\hline & & 0.664 & 0.655 & 0.545 & 0.516 & 0.539 & 0.511 & 0.413 & 0.398 \\
\hline & $(\bar{x} \pm \delta)$ & 0.658 & 0.645 & 0.539 & 0.527 & 0.515 & 0.503 & 0.408 & 0.401 \\
\hline & $(\%)$ & \pm 0.03 & \pm 0.009 & \pm 0.008 & \pm 0.01 & \pm 0.03 & \pm 0.01 & \pm 0.004 & \pm 0.007 \\
\hline \multirow{5}{*}{210} & & 0.706 & 0.680 & 0.575 & 0.570 & 0.512 & 0.549 & 0.436 & 0.435 \\
\hline & $\left(\mathrm{x}_{\mathrm{i}}\right)$ & 0.686 & 0.675 & 0.587 & 0.565 & 0.560 & 0.537 & 0.439 & 0.441 \\
\hline & & 0.698 & 0.691 & 0.586 & 0.581 & 0.578 & 0.531 & 0.445 & 0.420 \\
\hline & $(\bar{x} \pm \delta)$ & 0.697 & 0.682 & 0.583 & 0.572 & 0.550 & 0.539 & 0.437 & 0.432 \\
\hline & (\%) & \pm 0.01 & \pm 0.008 & \pm 0.006 & \pm 0.008 & \pm 0.03 & \pm 0.009 & \pm 0.01 & \pm 0.01 \\
\hline
\end{tabular}

Dependence of arithmetic mean values of water absorption of exposure time [specimen H.S. (a) and specimen I.R.S. (b)]

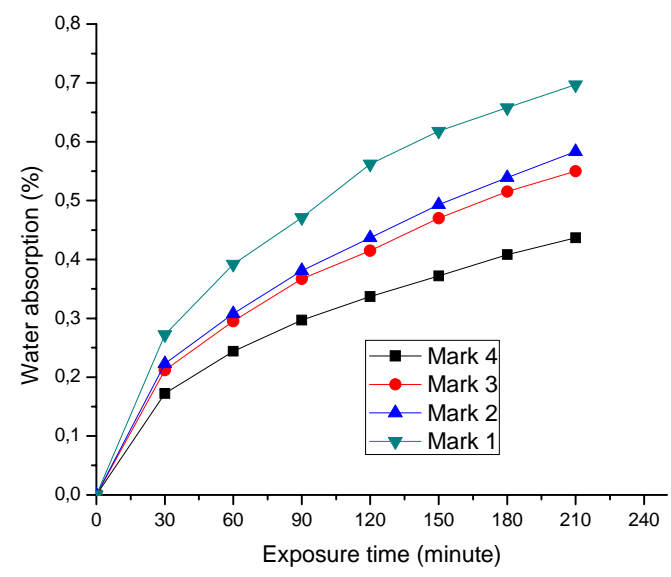

(a) for all four PR systems in boiling water for a period up to 210 minutes is presented in Fig. 15.

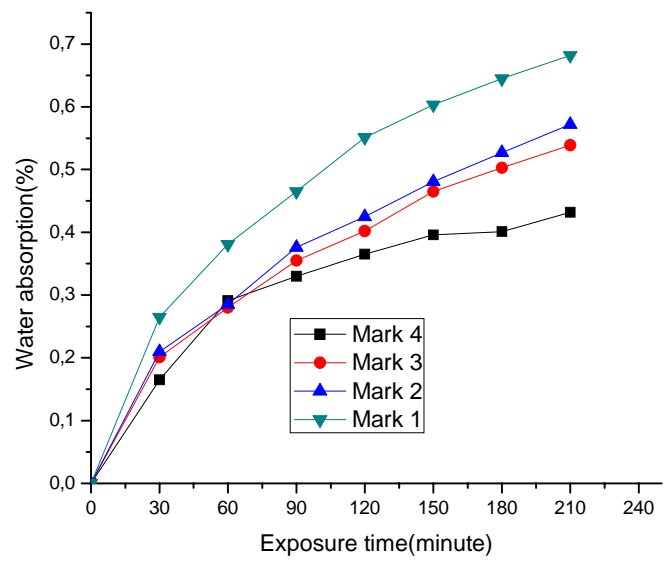

(b)

Figure 15. Dependence of arithmetic mean values of water absorption of exposure time [specimen H.S. (a) and specimen I.R.S. (b)] for all four PR systems in boiling water for a period up to 210 minutes

Specimens marks H.S. and I.R.S. of all four mentioned PR systems were immersed in boiling water for a period up to 210 minutes. Data regarding water soluble matter [single values $\left(\mathrm{x}_{\mathrm{i}}\right)$ and arithmetic mean value $\left.(\bar{x} \pm \delta)\right]$, recorded during this testing, are shown in Table 12.

Table 12. Water soluble matter [single values $\left(\mathrm{x}_{\mathrm{i}}\right)$ and arithmetic mean value $(\bar{x} \pm \delta)$ ] of specimens marks H.S. and I.R.S. of all four mentioned PR systems in boiling water for a period up to 210 minutes

\begin{tabular}{|c|c|c|c|c|c|c|c|c|c|}
\hline \multirow{3}{*}{\multicolumn{2}{|c|}{$\begin{array}{l}\text { Exposure time } \\
\text { (minute) }\end{array}$}} & \multicolumn{8}{|c|}{ PR system mark } \\
\hline & & \multicolumn{2}{|c|}{ Mark 1} & \multicolumn{2}{|c|}{ Mark 2} & \multicolumn{2}{|c|}{ Mark 3} & \multicolumn{2}{|c|}{ Mark 4} \\
\hline & & H.S. & I.R.S. & H.S. & I.R.S. & H.S. & I.R.S. & H.S. & I.R.S. \\
\hline \multirow{4}{*}{30} & & 0.463 & 0.470 & 0.328 & 0.314 & 0.307 & 0.290 & 0.238 & 0.235 \\
\hline & $\left(\mathrm{x}_{\mathrm{i}}\right)$ & 0.472 & 0.469 & 0.318 & 0.316 & 0.302 & 0.304 & 0.253 & 0.252 \\
\hline & & 0.478 & 0.456 & 0.326 & 0.330 & 0.294 & 0.291 & 0.256 & 0.239 \\
\hline & $\begin{array}{c}(\bar{x} \pm \delta) \\
(\%)\end{array}$ & $\begin{array}{c}0.471 \\
\pm 0.007\end{array}$ & $\begin{array}{c}0.465 \\
\pm 0.008\end{array}$ & $\begin{array}{c}0.324 \\
\pm 0.005\end{array}$ & $\begin{array}{c}0.320 \\
\pm 0.008\end{array}$ & $\begin{array}{c}0.301 \\
\pm 0.006\end{array}$ & $\begin{array}{c}0.295 \\
\pm 0.008\end{array}$ & $\begin{array}{c}0.249 \\
\pm 0.009\end{array}$ & $\begin{array}{c}0.242 \\
\pm 0.009\end{array}$ \\
\hline \multirow{5}{*}{60} & & 0.593 & 0.592 & 0.422 & 0.420 & 0.396 & 0.395 & 0.339 & 0.321 \\
\hline & $\left(\mathrm{X}_{\mathrm{i}}\right)$ & 0.592 & 0.594 & 0.433 & 0.421 & 0.408 & 0.393 & 0.328 & 0.324 \\
\hline & & 0.606 & 0.581 & 0.435 & 0.431 & 0.411 & 0.406 & 0.326 & 0.330 \\
\hline & $(\bar{x} \pm \delta)$ & 0.597 & 0.589 & 0.430 & 0.424 & 0.405 & 0.398 & 0.331 & 0.325 \\
\hline & $(\%)$ & \pm 0.008 & \pm 0.007 & \pm 0.007 & \pm 0.006 & \pm 0.008 & \pm 0.007 & \pm 0.007 & \pm 0.005 \\
\hline
\end{tabular}




\begin{tabular}{|c|c|c|c|c|c|c|c|c|c|}
\hline \multirow[t]{2}{*}{90} & $\begin{array}{l}\left(\mathrm{x}_{\mathrm{i}}\right) \\
(\%)\end{array}$ & $\begin{array}{l}0.682 \\
0.685 \\
0.676\end{array}$ & $\begin{array}{l}0.665 \\
0.670 \\
0.681\end{array}$ & $\begin{array}{l}0.495 \\
0.493 \\
0.485\end{array}$ & $\begin{array}{l}0.478 \\
0.480 \\
0.491 \\
\end{array}$ & $\begin{array}{l}0.475 \\
0.473 \\
0.465 \\
\end{array}$ & $\begin{array}{l}0.460 \\
0.462 \\
0.470 \\
\end{array}$ & $\begin{array}{l}0.399 \\
0.411 \\
0.414 \\
\end{array}$ & $\begin{array}{l}0.396 \\
0.399 \\
0.408 \\
\end{array}$ \\
\hline & $\begin{array}{c}(\bar{x} \pm \delta) \\
(\%)\end{array}$ & $\begin{array}{c}0.681 \\
\pm 0.004 \\
\end{array}$ & $\begin{array}{c}0.672 \\
\pm 0.008 \\
\end{array}$ & $\begin{array}{c}0.491 \\
\pm 0.005 \\
\end{array}$ & $\begin{array}{c}0.483 \\
\pm 0.007 \\
\end{array}$ & $\begin{array}{c}0.471 \\
\pm 0.005 \\
\end{array}$ & $\begin{array}{c}0.464 \\
\pm 0.005 \\
\end{array}$ & $\begin{array}{c}0.408 \\
\pm 0.008 \\
\end{array}$ & $\begin{array}{c}0.401 \\
\pm 0.006 \\
\end{array}$ \\
\hline \multirow[t]{2}{*}{120} & $\begin{array}{l}\left(\mathrm{x}_{\mathrm{i}}\right) \\
(\%)\end{array}$ & $\begin{array}{l}0.762 \\
0.764 \\
0.775 \\
\end{array}$ & $\begin{array}{l}0.760 \\
0.762 \\
0.746 \\
\end{array}$ & $\begin{array}{l}0.566 \\
0.569 \\
0.560 \\
\end{array}$ & $\begin{array}{l}0.560 \\
0.562 \\
0.546 \\
\end{array}$ & $\begin{array}{l}0.530 \\
0.531 \\
0.537\end{array}$ & $\begin{array}{l}0.521 \\
0.523 \\
0.534\end{array}$ & $\begin{array}{l}0.465 \\
0.466 \\
0.473 \\
\end{array}$ & $\begin{array}{l}0.455 \\
0.458 \\
0.467\end{array}$ \\
\hline & $\begin{array}{c}(\bar{x} \pm \delta) \\
(\%)\end{array}$ & $\begin{array}{c}0.767 \\
\pm 0.007\end{array}$ & $\begin{array}{c}0.756 \\
\pm 0.008\end{array}$ & $\begin{array}{c}0.565 \\
\pm 0.004\end{array}$ & $\begin{array}{c}0.556 \\
\pm 0.008\end{array}$ & $\begin{array}{c}0.533 \\
\pm 0.004\end{array}$ & $\begin{array}{c}0.526 \\
\pm 0.007\end{array}$ & $\begin{array}{c}0.468 \\
\pm 0.004\end{array}$ & $\begin{array}{c}0.460 \\
\pm 0.006\end{array}$ \\
\hline \multirow[t]{2}{*}{150} & $\begin{array}{l}\left(\mathrm{x}_{\mathrm{i}}\right) \\
(\%)\end{array}$ & $\begin{array}{l}0.830 \\
0.838 \\
0.828 \\
\end{array}$ & $\begin{array}{l}0.817 \\
0.815 \\
0.831 \\
\end{array}$ & $\begin{array}{l}0.630 \\
0.647 \\
0.634 \\
\end{array}$ & $\begin{array}{l}0.630 \\
0.621 \\
0.624 \\
\end{array}$ & $\begin{array}{l}0.600 \\
0.607 \\
0.596 \\
\end{array}$ & $\begin{array}{l}0.598 \\
0.596 \\
0.582 \\
\end{array}$ & $\begin{array}{l}0.542 \\
0.549 \\
0.544\end{array}$ & $\begin{array}{l}0.540 \\
0.539 \\
0.526 \\
\end{array}$ \\
\hline & $\begin{array}{c}(\bar{x} \pm \delta) \\
(\%)\end{array}$ & $\begin{array}{c}0.832 \\
\pm 0.005\end{array}$ & $\begin{array}{c}0.821 \\
\pm 0.008 \\
\end{array}$ & $\begin{array}{c}0.637 \\
\pm 0.009 \\
\end{array}$ & $\begin{array}{c}0.625 \\
\pm 0.005 \\
\end{array}$ & $\begin{array}{c}0.601 \\
\pm 0.005\end{array}$ & $\begin{array}{c}0.592 \\
\pm 0.008 \\
\end{array}$ & $\begin{array}{c}0.545 \\
\pm 0.003 \\
\end{array}$ & $\begin{array}{c}0.535 \\
\pm 0.008 \\
\end{array}$ \\
\hline \multirow[t]{2}{*}{180} & $\begin{array}{l}\left(\mathrm{x}_{\mathrm{i}}\right) \\
(\%)\end{array}$ & $\begin{array}{l}0.885 \\
0.888 \\
0.894 \\
\end{array}$ & $\begin{array}{l}0.870 \\
0.874 \\
0.884 \\
\end{array}$ & $\begin{array}{l}0.697 \\
0.695 \\
0.705\end{array}$ & $\begin{array}{l}0.695 \\
0.692 \\
0.680 \\
\end{array}$ & $\begin{array}{l}0.655 \\
0.648 \\
0.644 \\
\end{array}$ & $\begin{array}{l}0.634 \\
0.636 \\
0.650 \\
\end{array}$ & $\begin{array}{l}0.598 \\
0.608 \\
0.612 \\
\end{array}$ & $\begin{array}{l}0.600 \\
0.599 \\
0.583 \\
\end{array}$ \\
\hline & $\begin{array}{c}(\bar{x} \pm \delta) \\
(\%)\end{array}$ & $\begin{array}{c}0.889 \\
\pm 0.004 \\
\end{array}$ & $\begin{array}{l}0.877 \\
\pm 0.01 \\
\end{array}$ & $\begin{array}{c}0.699 \\
\pm 0.005 \\
\end{array}$ & $\begin{array}{c}0.689 \\
\pm 0.008 \\
\end{array}$ & $\begin{array}{c}0.649 \\
\pm 0.005 \\
\end{array}$ & $\begin{array}{c}0.640 \\
\pm 0.008 \\
\end{array}$ & $\begin{array}{c}0.606 \\
\pm 0.007 \\
\end{array}$ & $\begin{array}{c}0.594 \\
\pm 0.009 \\
\end{array}$ \\
\hline \multirow[t]{2}{*}{210} & $\begin{array}{l}\left(\mathrm{x}_{\mathrm{i}}\right) \\
(\%)\end{array}$ & $\begin{array}{l}0.922 \\
0.920 \\
0.944\end{array}$ & $\begin{array}{l}0.909 \\
0.908 \\
0.928\end{array}$ & $\begin{array}{l}0.752 \\
0.753 \\
0.765\end{array}$ & $\begin{array}{l}0.743 \\
0.744 \\
0.748\end{array}$ & $\begin{array}{l}0.726 \\
0.724 \\
0.734 \\
\end{array}$ & $\begin{array}{l}0.709 \\
0.711 \\
0.725 \\
\end{array}$ & $\begin{array}{l}0.630 \\
0.628 \\
0.641 \\
\end{array}$ & $\begin{array}{l}0.622 \\
0.615 \\
0.626 \\
\end{array}$ \\
\hline & $\begin{array}{c}(\bar{x} \pm \delta) \\
(\%)\end{array}$ & $\begin{array}{l}0.929 \\
\pm 0.01\end{array}$ & $\begin{array}{l}0.915 \\
\pm 0.01\end{array}$ & $\begin{array}{c}0.757 \\
\pm 0.007\end{array}$ & $\begin{array}{c}0.745 \\
\pm 0.003\end{array}$ & $\begin{array}{c}0.728 \\
\pm 0.005\end{array}$ & $\begin{array}{c}0.715 \\
\pm 0.008\end{array}$ & $\begin{array}{c}0.633 \\
\pm 0.007\end{array}$ & $\begin{array}{c}0.621 \\
\pm 0.005\end{array}$ \\
\hline
\end{tabular}

In Figure 16 are presented dependence of arithmetic mean values of water soluble matter of exposure time [specimen

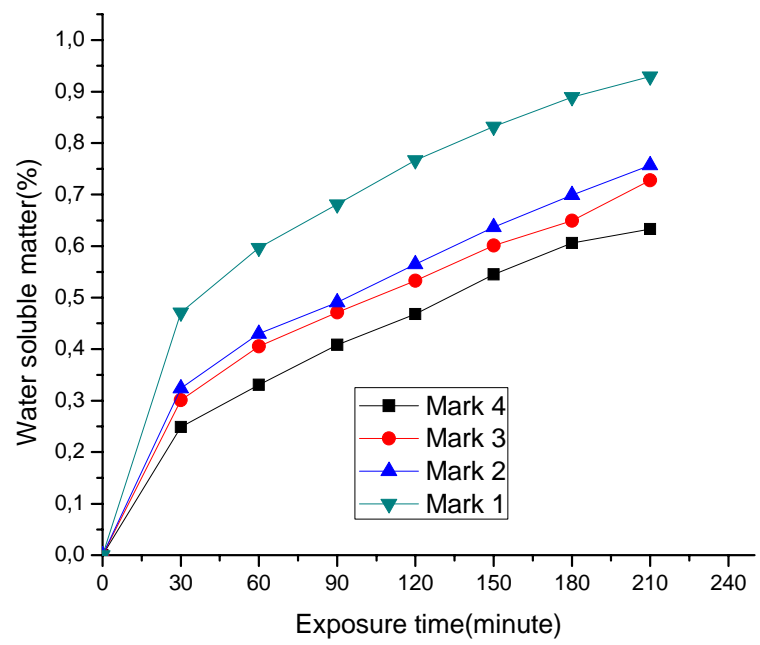

(a)
H.S. (a) and specimen I.R.S. (b)] for all four PR systems in boiling water for a period up to 210 minutes.

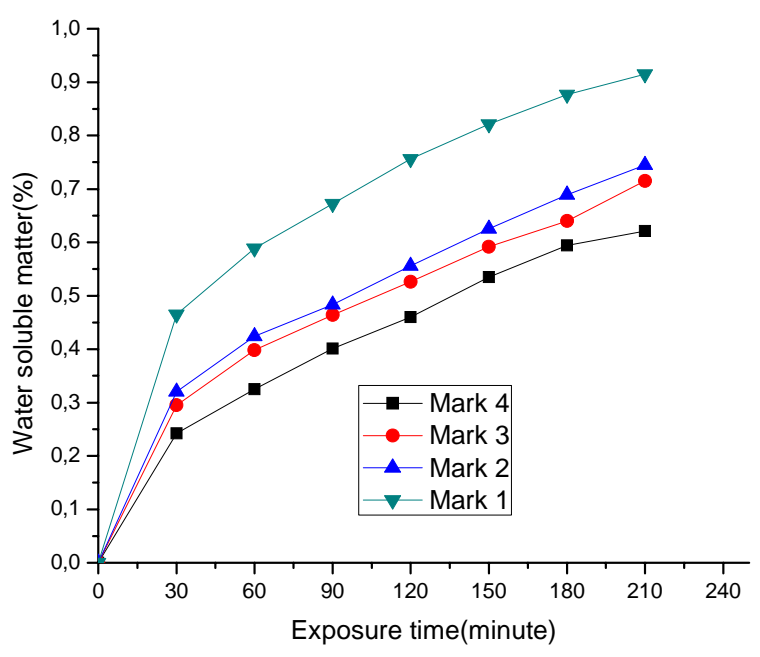

(b)

Figure 16. Dependence of arithmetic mean values of water soluble matter of exposure time [specimen H.S. (a) and specimen I.R.S. (b)] for all four PR systems in boiling water for a period up to 210 minutes

One specimen H.S. of each of four PR systems was immersed in boiling water for up to 210 minutes. Data recorded during drying these specimens to constant mass, after mentioned immersion, are presented in Table 13.

Table 13. Data recorded during drying one specimen H.S. of each of four PR systems to constant mass, after immersing in boiling water for a period up to 210 minutes

\begin{tabular}{|c|c|c|c|c||}
\hline \multirow{2}{*}{$\begin{array}{c}\text { Drying time } \\
\text { (hour) }\end{array}$} & \multicolumn{4}{|c|}{ PR system mark } \\
\cline { 2 - 5 } & Mark 1 & Mark 2 & Mark 3 & Mark 4 \\
\hline \hline 0 & 6.5561 & 6.3653 & 5.6541 & 5.8525 \\
\hline 24 & 6.5336 & 6.3420 & 5.6361 & 5.8365 \\
\hline 48 & 6.5175 & 6.3310 & 5.6248 & 5.8266 \\
\hline 72 & 6.5083 & 6.3255 & 5.6190 & 5.8189 \\
\hline 96 & 6.5025 & 6.3219 & 5.6159 & 5.8162 \\
\hline 120 & 6.4987 & 6.3200 & 5.6143 & 5.8152 \\
\hline
\end{tabular}

\begin{tabular}{||c|c|c|c|c||}
\hline 144 & 6.4970 & 6.3191 & 5.6135 & 5.8151 \\
\hline 168 & 6.4961 & 6.3190 & 6.6134 & $/$ \\
\hline 192 & 6.4960 & $/$ & $/$ & $/$ \\
\hline
\end{tabular}

Data in Table 13 are similar to those presented in Table 7 regarding drying time of specimen to constant mass.

PR system mark 1 showed the longest drying time of a specimen because this system had the highest both water absorption value and water soluble matter value.

PR system mark 4 has the smallest both water absorption value and water soluble matter value and the smallest drying time was necessary to obtain specimen of constant mass.

Barcol hardness of H.S. of each of four PR systems, [single values $\left(\mathrm{x}_{\mathrm{i}}\right)$ and adopted value for later analysis], expressed in Barcol degree, after immersing in boiling water for the periods up to 210 minutes, are presented in Table 14. 
Table 14. Barcol hardness of H.S. of each of four PR systems, after immersing in boiling water for the periods up to 210 minutes

\begin{tabular}{|c|c|c|c|c|c|c|c|c||}
\hline \multirow{2}{*}{$\begin{array}{c}\text { Exposure } \\
\text { time } \\
\text { (minute) }\end{array}$} & \multicolumn{9}{|c|}{ Mark 1 } & \multicolumn{2}{c|}{ Mark 2 } & \multicolumn{2}{c||}{ Mark 3 } & \multicolumn{3}{c||}{ Mark 4 } \\
\cline { 2 - 11 } & $\begin{array}{c}\text { Single } \\
\text { values } \\
\text { (B.D.) }\end{array}$ & $\begin{array}{c}\text { Adopted } \\
\text { value } \\
\text { (B.D.) }\end{array}$ & $\begin{array}{c}\text { Single } \\
\text { values } \\
\text { (B.D.) }\end{array}$ & $\begin{array}{c}\text { Adopted } \\
\text { value } \\
\text { (B.D.) }\end{array}$ & $\begin{array}{c}\text { Single } \\
\text { values } \\
\text { (B.D.) }\end{array}$ & $\begin{array}{c}\text { Adopted } \\
\text { value } \\
\text { (B.D.) }\end{array}$ & $\begin{array}{c}\text { Single } \\
\text { values } \\
\text { (B.D.) }\end{array}$ & $\begin{array}{c}\text { Adopted } \\
\text { value } \\
\text { (B.D.) }\end{array}$ \\
\hline \hline 0 & $42,41,42$ & 42 & $42,42,42$ & 42 & $44,44,43$ & 44 & $41,42,41$ & 41 \\
\hline 30 & $40,39,40$ & 39 & $42,41,41$ & 41 & $43,42,43$ & 43 & $41,40,40$ & 40 \\
\hline 60 & $38,37,37$ & 37 & $41,40,41$ & 41 & $42,42,42$ & 42 & $39,40,39$ & 39 \\
\hline 90 & $35,36,35$ & 35 & $40,39,40$ & 40 & $42,41,42$ & 42 & $38,38,39$ & 38 \\
\hline 120 & $33,34,33$ & 33 & $39,38,39$ & 39 & $40,41,40$ & 40 & $37,38,37$ & 37 \\
\hline 150 & $32,33,32$ & 32 & $38,38,37$ & 38 & $40,39,40$ & 40 & $36,36,36$, & 36 \\
\hline 180 & $31,32,31$ & 31 & $37,36,37$ & 37 & $39,38,39$ & 39 & $35,34,35$ & 35 \\
\hline 210 & $30,31,30$ & 30 & $36,35,36$ & 36 & $38,37,38$ & 38 & $34,35,34$ & 34 \\
\hline
\end{tabular}

Barcol hardness (adopted values) of H.S. of four PR systems, after immersing in boiling water for the periods up to the 210 minutes, are presented in Fig. 17.

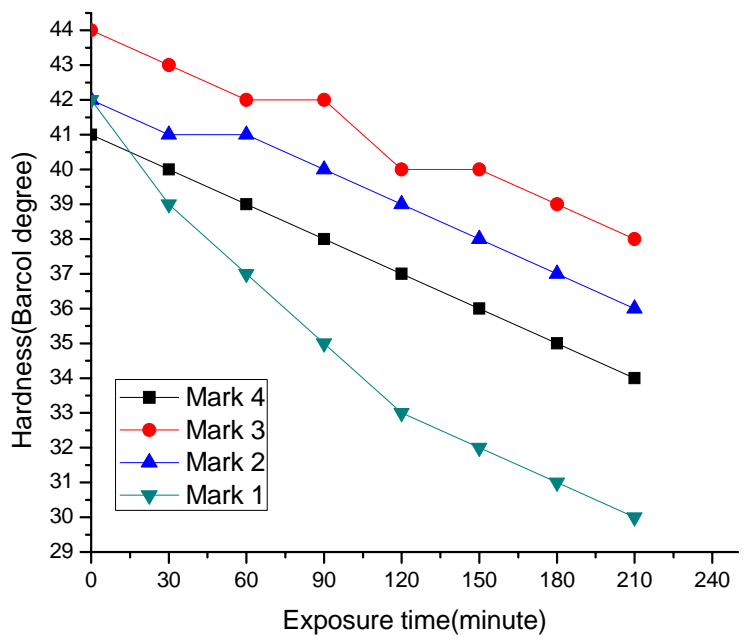

Figure 17. Barcol hardness (adopted values) of H.S. of four PR systems, after immersing in boiling water for the periods up to the 210 minutes

Changes in Barcol hardness after immersion in boiling water are similar to those after the influence of distillated and synthetic sea water, but in greater extent. PS systems mark 2 and 3 have similar drop of Barcol hardness (about $13 \%$ ) and PS system mark 4 had something higher decreasing of this characteristics (about $17 \%$ ). The highest drop of this property is recorded at PS system mark 1 (about $30 \%$ ) and it is important to emphasized that Barcol hardness after immersing in boiling water for the period of the 210 minutes is at the lowest acceptable level for correctly manufactured polyester resin product (30 Barcol degrees).

Specimens I.R.S. of each of four PR systems were immersed in boiling water for a period up to 210 minutes. Impact resistance [single values $\left(\mathrm{x}_{\mathrm{i}}\right)$ and arithmetic mean value $(\bar{x} \pm \delta)]$ of these specimens, after mentioned immersion, are presented in Table 15.

Table 15. Impact resistance of I.R.S. of each of four PR systems, after immersing in boiling water for periods up to 210 minutes

\begin{tabular}{|c|c|c|c|c|c|}
\hline \multirow{2}{*}{\multicolumn{2}{|c|}{$\begin{array}{l}\text { Exposure } \\
\text { time } \\
\text { (minute) }\end{array}$}} & \multicolumn{4}{|c|}{ PR system mark } \\
\hline & & Mark 1 & Mark 2 & Mark 3 & Mark 4 \\
\hline \multirow{2}{*}{0} & $\begin{array}{c}\left(x_{\mathrm{i}}\right) \\
\left(\mathrm{kJ} / \mathrm{m}^{2}\right)\end{array}$ & $\begin{array}{c}5,00 ; 5,21 ; \\
5,12\end{array}$ & $\begin{array}{c}17,02 ; 16,83 ; \\
16,54\end{array}$ & $\begin{array}{c}7,44 ; 6,81 ; \\
6,72\end{array}$ & $\begin{array}{c}14,62 ; 14,91 ; \\
15,22\end{array}$ \\
\hline & $\begin{array}{l}(\bar{x} \pm \delta) \\
\left(\mathrm{kJ} / \mathrm{m}^{2}\right)\end{array}$ & $5,11 \pm 0,10$ & $16,79 \pm 0,24$ & $6,99 \pm 0,39$ & $14,92 \pm 0,30$ \\
\hline
\end{tabular}

\begin{tabular}{|c|c|c|c|c|c|}
\hline \multirow{2}{*}{30} & $\begin{array}{c}\left(\begin{array}{c}\left(x_{\mathrm{i}}\right) \\
\left(\mathrm{kJ} / \mathrm{m}^{2}\right)\end{array}\right. \\
\end{array}$ & $\begin{array}{c}4,51 ; 5,30 \\
4,62 \\
\end{array}$ & \begin{tabular}{|c|}
15,$53 ; 16,22 ;$ \\
16,61 \\
\end{tabular} & $\begin{array}{c}6,59 ; 6,90 \\
6,91 \\
\end{array}$ & $\begin{array}{c}13,91 ; 14,82 ; \\
13,61\end{array}$ \\
\hline & $\begin{array}{l}(\bar{x} \pm \delta) \\
\left(\mathrm{kJ} / \mathrm{m}^{2}\right)\end{array}$ & $4,81 \pm 0,43$ & $16,129 \pm 0,55$ & $6,80 \pm 0,18$ & $14,11 \pm 0,63$ \\
\hline \multirow{2}{*}{60} & $\begin{array}{c}\left(x_{\mathrm{i}}\right) \\
\left(\mathrm{kJ} / \mathrm{m}^{2}\right)\end{array}$ & $\begin{array}{c}4,73 ; 4,31 ; \\
4,50\end{array}$ & $\begin{array}{c}15,01 ; 16,11 \\
15,72\end{array}$ & $\begin{array}{c}6,51 ; 6,90 \\
6,42\end{array}$ & $\begin{array}{c}14,02 ; 12,71 ; \\
13,12\end{array}$ \\
\hline & $\begin{array}{l}(\bar{x} \pm \delta) \\
\left(\mathrm{kJ} / \mathrm{m}^{2}\right)\end{array}$ & $4,45 \pm 0,31$ & $15,61 \pm 0,24$ & $6,61 \pm 0,25$ & $13,55 \pm 0,73$ \\
\hline \multirow[t]{2}{*}{9} & $\begin{array}{c}(\mathrm{xi}) \\
\left(\mathrm{kJ} / \mathrm{m}^{2}\right)\end{array}$ & $\begin{array}{c}4,05 ; 4,51 ; \\
4,22\end{array}$ & $\begin{array}{c}14,22 ; 15,21 \\
15,30\end{array}$ & $\begin{array}{c}6,26 ; 6,71 ; \\
6,18 \\
\end{array}$ & $\begin{array}{c}13,42 ; 12,11 ; \\
13,22\end{array}$ \\
\hline & $\begin{array}{l}(\bar{x} \pm \delta) \\
\left(\mathrm{kJ} / \mathrm{m}^{2}\right)\end{array}$ & $4,26 \pm 0,23$ & $14,91 \pm 0,60$ & $6,38 \pm 0,28$ & $12,92 \pm 0,70$ \\
\hline & $\begin{array}{c}\left(x_{\mathrm{i}}\right) \\
\left(\mathrm{kJ} / \mathrm{m}^{2}\right)\end{array}$ & $\begin{array}{c}3,51 ; 3,88 \\
4,51\end{array}$ & $\begin{array}{c}15,23 ; 14,09 \\
13,90\end{array}$ & $\begin{array}{c}5,50 ; 5,91 \\
6,28\end{array}$ & $\begin{array}{c}13,01 ; 12,02 ; \\
12,81\end{array}$ \\
\hline & $\begin{array}{l}(\bar{x} \pm \delta) \\
\left(\mathrm{kJ} / \mathrm{m}^{2}\right)\end{array}$ & $3,97 \pm 0,51$ & $14,41 \pm 0,72$ & $5,89 \pm 0,39$ & $12,61 \pm 0,52$ \\
\hline \multirow{2}{*}{150} & $\begin{array}{c}\left(x_{\mathrm{i}}\right) \\
\left(\mathrm{kJ} / \mathrm{m}^{2}\right)\end{array}$ & $\begin{array}{c}3,21 ; 3,91 ; 3, \\
22\end{array}$ & $\begin{array}{c}13,71 ; 14,82 ; 13 \\
, 48\end{array}$ & 5,$41 ; 6,04 ; 5,72$ & $\begin{array}{c}11,91 ; 11,52 ; 13,0 \\
2\end{array}$ \\
\hline & $\begin{array}{l}(\bar{x} \pm \delta) \\
\left(\mathrm{kJ} / \mathrm{m}^{2}\right)\end{array}$ & $3,45 \pm 0,41$ & $14,00 \pm 0,72$ & $5,73 \pm 0,32$ & $12,15 \pm 0,78$ \\
\hline \multirow{2}{*}{180} & $\begin{array}{c}\left(x_{\mathrm{i}}\right) \\
\left(\mathrm{kJ} / \mathrm{m}^{2}\right)\end{array}$ & $\begin{array}{c}2,84 ; 3,32 ; 3, \\
03\end{array}$ & $\begin{array}{c}13,01 ; 13,12 ; 14 \\
, 13\end{array}$ & 5,$42 ; 5,88 ; 5,35$ & $\begin{array}{c}12,01 ; 11,02 ; 11,8 \\
1\end{array}$ \\
\hline & $\begin{array}{l}(\bar{x} \pm \delta) \\
\left(\mathrm{kJ} / \mathrm{m}^{2}\right)\end{array}$ & $3,06 \pm 0,24$ & $13,42 \pm 0,60$ & $6,38 \pm 0,28$ & $11,61 \pm 0,51$ \\
\hline \multirow{2}{*}{210} & $\begin{array}{c}\left(x_{\mathrm{i}}\right) \\
\left(\mathrm{kJ} / \mathrm{m}^{2}\right)\end{array}$ & $\begin{array}{c}2,41 ; 2,52 ; 2 \\
68\end{array}$ & $\begin{array}{c}13,02 ; 12,66 ; 14 \\
, 12\end{array}$ & 5,$28 ; 5,89 ; 5,10$ & $\begin{array}{c}11,04 ; 10,63 ; 12,1 \\
2\end{array}$ \\
\hline & $\begin{array}{l}(\bar{x} \pm \delta) \\
\left(\mathrm{kJ} / \mathrm{m}^{2}\right)\end{array}$ & $2,54 \pm 0,14$ & $13,23 \pm 0,70$ & $5,42 \pm 0,28$ & $11,25 \pm 0,77$ \\
\hline
\end{tabular}

Impact resistance of I.R.S. of four PR systems, after immersing in boiling water for the period up to the 210 minutes, is presented in Fig. 18.

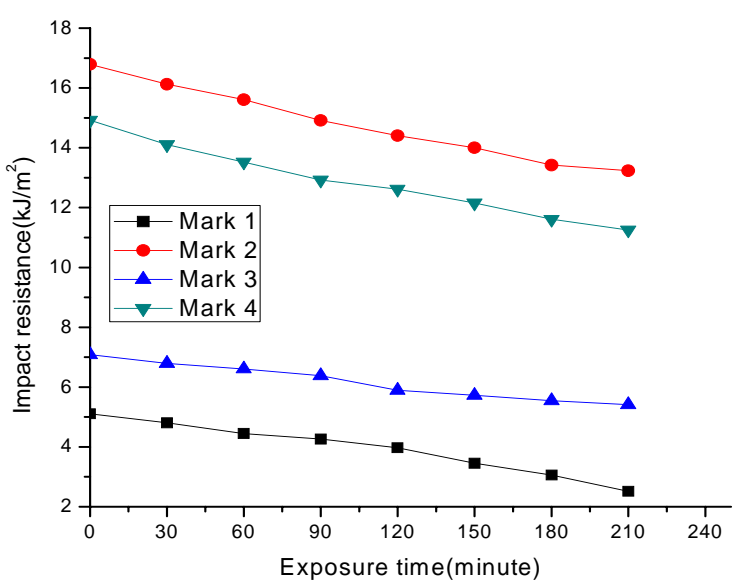

Figure 18. Impact resistance of I.R.S. of four PR systems, after immersing in boiling water for the periods up to the 210 minutes 
After exposure to severe conditions in boiling water for 210 minutes PR system mark 2 and PR system mark 3 showed the smallest, mutual almost the same, diminution of impact resistance (about $20 \%$ ). Somewhat higher decrease of this characteristic was observed at PR system 4 (about $40 \%$ ). The highest reduction of impact resistance was recorded at PR system mark 1 (about $60 \%$ ), and this fact represents another argument that this system has higher sensitivity to water, especially boiling.

Similar results of water absorption and water soluble matter in boiling distillated water, obtained by testing hardness specimens and impact resistance specimens (Tables 10 to 15 and Figures 13 to 18) pointed out that procedures for producing and investigating of both specimens have been done correctly.

\section{Conclusions}

From the all presented statements and results, it can be concluded:

1. Based on the composition and obtained laboratory results of polyester resin system NPG/IPA/AMK/St, it can be concluded that neopentyl glycol has a positive effect on tested physical and mechanical characteristics.

2. Polyester resin system DEG/PPG/APA/AMK/St showed the smallest water resistance. Analyzing the composition of the mentioned system, this fact can be attributed to the sensitivity of ether linkages of diethylene glycol, primarily, and to a smaller molecular mass, regarding the orthophthalic acid.

3. Branched alcohols have a positive influence on impact resistance of tested polyester resin systems as well as high molecular weight.

4. All four polyester resin systems have similar initial Barcol hardness and, excluded polyester resin system DEG/PPG/APA/AMK/St, showed approximately similar behavior in tested environments.

5. Boiling water caused higher drop of tested properties of used polyester resin systems than distillated and synthetic sea water at $23^{\circ} \mathrm{C}$. This is extremely obvious regarding the Barcol hardness of polyester resin system DEG/PPG/APA/AMK/St.

6. Synthetic sea water has slightly less effects on tested physical and mechanical characteristics of four cured polyester resin systems than distillated water.

\section{References}

[1] RADUlOVIĆ,J., ŽEGARAC,N.,: Internally Stressed Polymeric Composite/Metal Product: Manufacturing, Testing And Quality Verifying By Simplified Procedure, Scientific Technical Review, ISSN 1820-0206, 2017, Vol.67, No.1, pp.45-53.

[2] LAWRENCE,R.J.: Polyester Resin, Reinhold Publishing Corporation, New York, 1990.

[3] WEATHERHEAD,R.G.: FRP Technology, Fibre Reinforced Resin Systems, Applied Science Publishers LTD, London, 1980.

[4] Nomenclature of Organic Chemistry: IUPAC Recommendations and Preferred Names 2013 (Blue Book). Cambridge: The Royal Society of Chemistry, 2014, doi:10.1039/9781849733069-FP001. ISBN 978-085404-182-4.
[5] MARRAFFA,J.M., HOLLAND,M.G., STORK,C.M., HOY,C.D., HODGMAN,M.J.: Diethylene Glycol: Widely Used Solvent Presents Serious Poisoning Potential, J. Emerg Med., 2008, Vol.35, No.4, pp.401-406.

[6] WEISSERMEL,K., ARPE,H.J., LINDLEY,C.R.: Industrial Organic Chemistry (4 ed.), Wiley-VCH, 2003, pp.214-215, ISBN 978-3-52730578-0, retrieved 2009-07-20.

[7] PRITCHARD,G.: Development in Reinforced Plastics-1, Resin Matrix Aspects, Applied Science Publishers, London, 1980.

[8] JAYET,Y., SAINT-PERRE,N., TATIBOUET,J., ZELLOUF,D.: Monitoring the hydrolytic degradation of composites by a piezoelectric method, Ultrasonics, 1996, 34, pp.397-400.

[9] DAVIS,A., SIMS,D.: Weathering of Polymers, Applied Science Publishers, London and New York, 1983.

[10] RADULOVIĆ,J.: Thin Wall and Thick Wall Filament Wound Polymeric Composite Tubes: Mechanical Characteristics Caused by Internal Hydraulic Pressure, Scientific Technical Review, ISSN 18200206, 2013,Vol.63, No.1, pp.63-69.

[11] A model specification for FRP composites for civil engineering structures (fiber reinforced polymer composite materials). Construction and Building Materials. 2003-09-01. http://www.accessmylibrary.com/coms2/summary_028619509666_ITM. Retrieved 2008-06-15.

[12] ECKSTEIN,B.H.: ACS Org. Coatings Div. Plast. Chem. Preprints, 1978, 38, p 503.

[13] PRITCHARD,G., SPEAKE,S.D.: The use of water absorption kinetic data to predict laminate property changes, Composites, July 1987, Vol.18, pp.227-232.

[14] BELAN,F., BELLENGER,V., MORTAIGNE,B., VERDU,J.: Water swelling of polyester networks, Macromolecular Symposia, April 1995, Vol.93, No.1, pp. 81-88, https://doi.org/10.1002/masy.19950930112.

[15] PRITCHARD,G.: Development in Reinforced Plastics-2, Properties of Laminates, Applies Science Publishers, London, 1982.

[16] ROSATO,D.V.: Reinforced Plastics Handbook, 3rd ed., Elsevier Advanced Technology Ltd., Oxford, 2004, ISBN 1-8561-74506.

[17] RADULOVIĆ,J., ČITAKOVIĆ,S.: Mechanical Characteristics of Joints Between Glass Fiber Reinforced Composite Tubes and Steel Cylinder Obtained by Different Fastening Elements, Scientific Technical Review, ISSN 1820-0206, 2016, Vol.66, No.2, pp.28-35.

[18] https://ihsmarkit.com/products/unsaturated-polyester-resins-chemicaleconomics-handbook.html.

[19] NONVEILLER,F.: Jugoslavenski registar brodova, PRAVILNIK O MATERIJALIMA I ELEKTRIČNOM ZAVARIVANJU, Deo I, Materijali, NITTP "Slobodna Dalmacija", Split, YUGOSLAVIA, 1977.

[20] ISO 62 Plastics-Determination of water absorption,ISO,Case postal 56,CH-1211 Geneva 20, Switzerland, 2016.

[21] ISO 179-1, Plastics - Determination of Charpy impact properties - Part 1: Non-instrumented impact test, ISO (the International Organization for Standardization), Case postal 56, CH-1211 Geneva 20, Switzerland, 2010.

[22] ASTM D 2583-13a, Standard Test Method for Indentation Hardness of Rigid Plastics by Means of a Barcol Impressor, ASTM International, West Conshohocken, PA, 2013, www.astm.org.

[23] ASTM D1200-10(2014), Standard Test Method for Viscosity by Ford Viscosity Cup, ASTM International, West Conshohocken, PA, 2014, www.astm.org.

[24] ISO 2114 - Plastics (PRs) and paints and varnishes (binders) Determination of partial acid value and total acid value, ISO, Case postal 56, CH-1211 Geneva 20, Switzerland, 2000.

[25] ISO 2535:-3), Plastics - Unsaturated-polyester resins - Measurement of gel time at ambient temperature. ISO, Case postal 56, CH-1211 Geneva 20, Switzerland, 2001. 


\title{
Uticaj sastava poliesterskih smola, destilovane i sintetičke morske vode i povišene temperature na mehaničke i fizičke karakteristike četiri očvrsla poliesterska sistema
}

\begin{abstract}
U ovom članku je prezentovan uticaj sastava smole i destilovane i sintetičke morske vode na mehaničke i fizičke karakteristike četiri različita poliesterska sistema. Prikazani su osnovni podaci o hemiji poliesterskih smola, sastojaka sistema poliesterskih smola (višehidroksilni alkoholi, nezasićene višebazne kiseline, zasićene višebazne kiseline i monomer), procesu izrade očvrslih $i$ naknadno umreženih uzoraka i proceduri ispitivanja. Uzorci četiri sistema poliesterskih sistema bili su izloženi uticaju destilovane i sintetičke morske vode temperature laboratorije $\left(23^{\circ} \mathrm{C}\right)$ za period od trideset dana (prvi eksperiment) $\mathrm{i}$ destilovane ključale vode $\left(100^{\circ} \mathrm{C}\right)$ za period od 210 minuta (drugi eksperiment). Tvrdoća, udarna žilavost, vodorastvorljive materije i upijanje vode uzoraka četiri sistema poliesterskih smola su zabeleženi kao parametri koji pokazuju promenu kvaliteta ispitivanih materijala u pomenutim uslovima u oba eksperimenta. Utvrđeno je da sastojci i destilovana i sintetička morska voda imaju značajan uticaj na ispitivane mehaničke i fizičke karakteristike četiri sistema poliesterskih smola.
\end{abstract}

Ključne reči: poliesterske smole, mehaničke karakteristike, fizičke osobine, destilovana voda, morska voda, sintetička voda, rezultati ispitivanja.

\section{Влияние состава полиэфирных смол, дистиллированной и синтетической морской воды и повышенной температуры на механические и физические характеристики четырёх отверждённых полиэфирных систем}

\begin{abstract}
В этой статье представлено влияние состава смолы и дистиллированной и синтетической морскых вод на механические и физические характеристики четырёх различных полиэфирных систем. Представлены основные данные о химии полиэфирных смол, ингредиентов системы полиэфирных смол (многоатомных спиртов, ненасыщенных многоатомных кислот, насыщенных многоосноных кислот и мономеров), о процессе изготовления отверждённых и впоследствии сетевых образцов и о методике испытаний. Образцы четырёх систем полиэфирных систем подвергались воздействию дистиллированной и синтетической морскых вод при лабораторной температуре $\left(23^{\circ} \mathrm{C}\right)$ в течение тридцати дней (первый эксперимент) и дистиллированной кипячённой воды $\left(100^{\circ} \mathrm{C}\right)$ в течение 210 минутного периода (второй эксперимент). Твёрдость, ударопрочность, водорастворимые вещества и водопоглощение образцов четырёх систем полиэфирных смол были записаны в качестве параметров, которые показывают изменение качества испытуемых материалов в указанных условиях в обоих экспериментах. Было установлено, что ингредиенты и дистиллированная и синтетическая морскые воды оказывают существенное влияние на исследуемые механические и физические характеристики четырёх систем полиэфирных смол.
\end{abstract}

Ключевые слова: полиэфирные смолы, механические свойства, физические свойства, дистиллированная вода, морская вода, синтетическая вода, результаты испытаний.

\section{Influence de la composition des résines polyester, de l'eau de mer distillée et synthétique et la température élevée sur les caractéristiques mécaniques et physiques de quatre systèmes durcis polyester}

\begin{abstract}
Dans ce papier on présente l'influence de la composition de la résine et de l'eau de mer distillée et synthétique sur les caractéristiques mécaniques et physiques pour quatre différents systèmes de polyester. Les données basiques sur la chimie des résines polyester, les composantes des systèmes de ces résines (alcools polyhydriques, acides polybasiques non saturées ou saturées et monomère), processus de fabrication des échantillons durcis et des échantillons durcis postérieurement ainsi que la procédure de l'examen. Les échantillons des quatre systèmes polyester ont été exposés à l'influence de l'eau de mer distillée et synthétique à la température de laboratoire $\left(23^{\circ} \mathrm{C}\right)$ pendant trente jours (premier test) et à l'eau distillée bouillie $\left(100^{\circ} \mathrm{C}\right)$ pendant 210 minutes (second test). La dureté, la résistance à l'impact, les matières solubles dans l'eau et l'absorption de l'eau des échantillons des quatre systèmes des résines polyester ont été notés comme paramètres indiquant le changement de la qualité des matières examinées dans les conditions citées pour les deux examens. On a constaté que les composantes et l'eau distillée et synthétique avaient l'influence considérable sur les caractéristiques mécaniques et physiques chez les quatre systèmes des résines polyester.
\end{abstract}

